\title{
étude expérimentale d'un pieu soumis aux poussées latérales du sol
}

\author{
par \\ G. Bigot \\ Ingénieur au Laboratoire Régional de l'Est Parisien \\ F. Bourges \\ R. Frank \\ Ingénieurs au Laboratoire Central des Ponts et Chaussées
}

\section{Introduction}

L'expérimentation en vraie grandeur effectuée à Provins, en 1974-75, avait pour objectif l'observation du comportement d'un pieu libre, mis en place au pied d'un talus de remblai, et soumis aux poussées latérales d'une couche de sol mou.

Une première interprétation des résultats de cette expérimentation a fait l'objet d'une communication au IX Congrès International de Mécanique des Sols à Tokyo (1977) [1]

L'interprétation a été reprise, sur la base des récentes recommandations des Laboratoires des Ponts et Chaussées concernant le calcul des efforts engendrés dans les pieux par les poussées latérales du sol [2]. Cette nouvelle interprétation fait l'objet du présent article.

Seul un bref aperçu sur les conditions de l'expérimen- tation est donné ici. Les lecteurs intéressés par plus de détails pourront se reporter au rapport de recherches [3].

\section{Conditions de l'expérimentation}

\subsection{Le site}

II s'agit de la vallée de la Voulzie, franchie en remblai par la déviation de la R.N. 19 à Provins. Le pieu expérimental est placé en pied du talus de remblai. Dans le profil où il est situé, (fig. 1), le sol est constitué d'une couche de limons tourbeux et de tourbe de $11 \mathrm{~m}$ d'épaisseur environ, surmontée de 3 à $4 \mathrm{~m}$ de limons et d'argile plus ou moins organiques (tableau $n^{\circ} 1$ ). La craie est atteinte à environ $17 \mathrm{~m}$ de profondeur, et elle est séparée de la couche tourbeuse par 2 à $3 \mathrm{~m}$ de sables, graviers, et silex.

Tableau $n^{\circ} 1$

Caractéristiques moyennes des deux couches compressibles à l'emplacement du pieu.

\begin{tabular}{|c|c|c|c|c|c|c|c|c|}
\hline \multirow[b]{2}{*}{ Couche } & \multicolumn{3}{|c|}{ Identification } & \multirow{2}{*}{$\begin{array}{c}\text { Cohésion } \\
\text { non } \\
\text { drainée }\end{array}$} & \multicolumn{2}{|c|}{ - Compressibilité } & \multicolumn{2}{|c|}{ Pressiomètre } \\
\hline & $\begin{array}{c}\text { teneur } \\
\text { en } \\
\text { eau } \\
\text { w } \%\end{array}$ & $\begin{array}{c}\text { poids } \\
\text { volumique } \\
\text { sec } \\
y d \\
\text { en } \mathrm{kN} / \mathrm{m}^{3}\end{array}$ & $\begin{array}{c}\text { teneur } \\
\text { en } \\
\text { matières } \\
\text { organiques } \\
\%\end{array}$ & & $\begin{array}{c}C_{c} \\
1+e_{0}\end{array}$ & $\begin{array}{c}\mathrm{c}_{\mathrm{v}} \\
\text { en } \mathrm{cm}^{2} / \mathrm{s}\end{array}$ & $\begin{array}{l}\text { pression } \\
\text { limite } \\
\mathrm{p}_{e} \\
\text { en } \mathrm{kPa}\end{array}$ & $\begin{array}{c}\text { module } \\
\mathrm{E}_{\mathrm{M}} \\
\mathrm{en} \\
\mathrm{kPa}\end{array}$ \\
\hline $\begin{array}{l}0 \text { à } 4 \mathrm{~m} \\
\text { limons et } \\
\text { argiles }\end{array}$ & 30 & 14 & 10 & 40 & 0,14 & $6 \cdot 10^{-3}$ & 160 & 1100 \\
\hline $\begin{array}{c}4 \mathrm{~m} \text { à } 14,50 \mathrm{~m} \\
\text { limons tourbeux } \\
\text { et tourbe }\end{array}$ & 200 & 4 & 20 & 50 & 0,33 & $6 \cdot 10^{-3}$ & 250 & 1070 \\
\hline
\end{tabular}




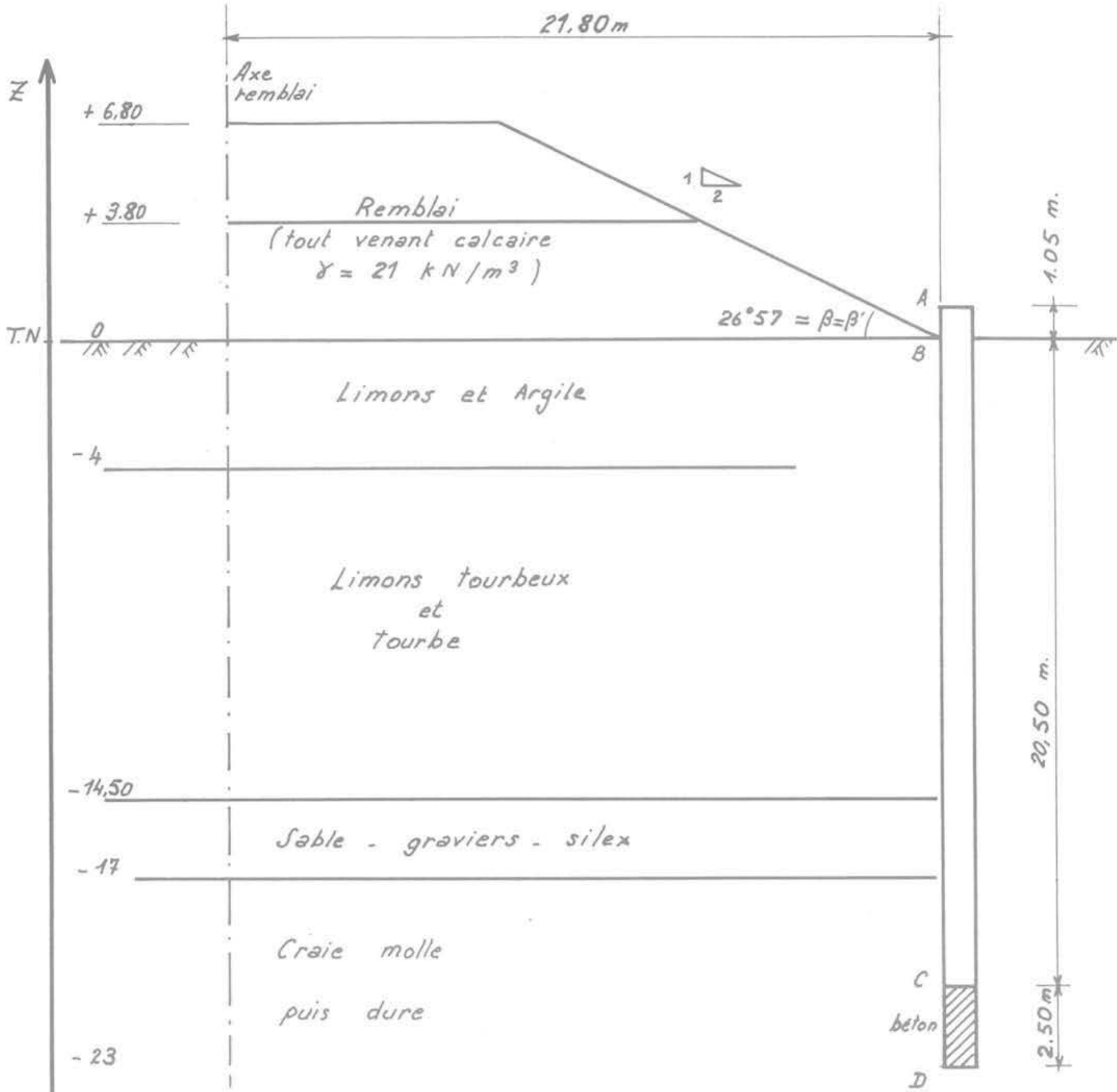

Fig. 1 Pieu et remblai de Provins - Schéma général

Parmi les essais pressiométriques effectués, on a retenu, pour l'interprétation, les essais suivants :

- essai au pressiomètre Menard, $\mathrm{P}_{1}$, réalisé à $5 \mathrm{~m}$ de l'axe du pieu (fig. 2);

- essai au pressiomètre autoforeur (P.A.F.) réalisé dans la zone d'essai, jusqu'à $13 \mathrm{~m}$ de profondeur, conformément aux règles actuelles de l'essai au P.A.F. Les résultats de cet essai effectué à posteriori (1977) sont donnés dans le tableau $n^{\circ} 4$.

\subsection{Le déroulement de l'expérience}

L'expérimentation a comporté 4 phases principales :

- phase 0 (avril 1974).

Réalisation d'un essai de chargement horizontal en tête. La charge est appliquée par paliers de $20 \mathrm{kN}$ maintenus 1 heure, jusqu'à $120 \mathrm{kN}$, puis le pieu est déchargé par paliers de $20 \mathrm{kN}$ maintenus une demi-heure.
- phase 1 ( $1^{\text {or }}$ juin-18 juillet 1974). Montée progressive du remblai jusqu'à $3,80 \mathrm{~m}$.

- phase 2 (18 juillet-30 juillet 1974).

Achèvement du remblaiement jusqu'à une hauteur de $6,80 \mathrm{~m}$.

- phase 3 (30 juillet-29 octobre 1974).

Phase de consolidation du sous-sol, le remblai restant à $6,80 \mathrm{~m}$. A la fin de cette phase, le tassement dans l'axe du remblai atteint $1,72 \mathrm{~m}$.

\subsection{Le dispositif expérimental (fig. 3)}

Le pieu visitable est constitué de 2 viroles en acier de $0,926 \mathrm{~m}$ de diamètre et de $12 \mathrm{~m}$ de longueur soudées bout à bout. II a été mis en place dans le sol, 5 mois avant la phase 0 , par vibrofonçage, en s'assurant que le sol remontait bien à l'intérieur et n'était pas refoulé. Une fois en place, il a été procédé au curage, coulage 


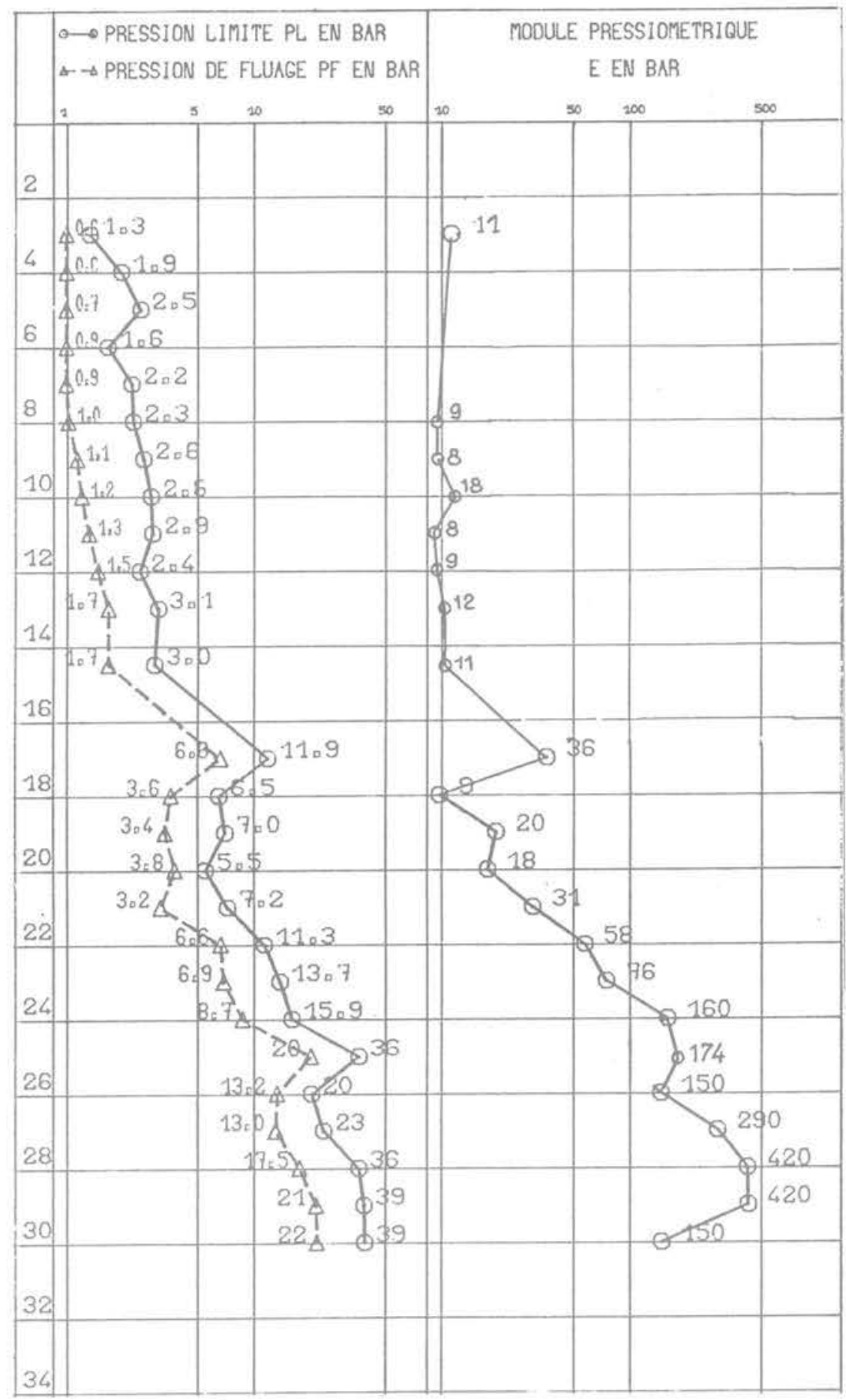

Fig. 2 Sondage pressiométrique P1

sous l'eau d'un bouchon de béton armé de 2,50 m d'épaisseur à la base, épuisement par pompage, puis soudage d'une plaque d'acier de $15 \mathrm{~mm}$ au-dessus du bouchon de béton. Ses caractéristiques moyennes sont données dans le tableau $n^{\circ} 2$.

\section{Mesures des efforts dans le pieu}

Les efforts dans le pieu (moments fléchissants) sont déterminés à partir des mesures d'élongation sur le pieu lui-même. Pour ces mesures, on a utilisé deux types d'appareillage.

1 Les jauges à fils résistants qui donnent des déformations quasiment ponctuelles : 50 jauges ont été mises en place sur le pieu.
Tableau $n^{\circ} 2$

Caractéristiques moyennes du pieu

\begin{tabular}{|l|l|}
\hline Diamètre extérieur & $\mathrm{B}=0,926 \mathrm{~m}$ \\
\hline Épaisseur d'acier & $\mathrm{e}=0,015 \mathrm{~m}$ \\
\hline Section d'acier & $\mathrm{s}=0,0429 \mathrm{~m}^{2}$ \\
\hline Moment d'inertie & $\mathrm{I}=4,45 \cdot 10^{-3} \mathrm{~m}^{4}$ \\
\hline Module d'Young de l'acier & $\mathrm{E}=2 \cdot 10^{8} \mathrm{kPa}$ \\
\hline & $\mathrm{El}=8,9 \cdot 10^{5} \cdot \mathrm{kN} \times \mathrm{m}^{2}$ \\
\hline
\end{tabular}




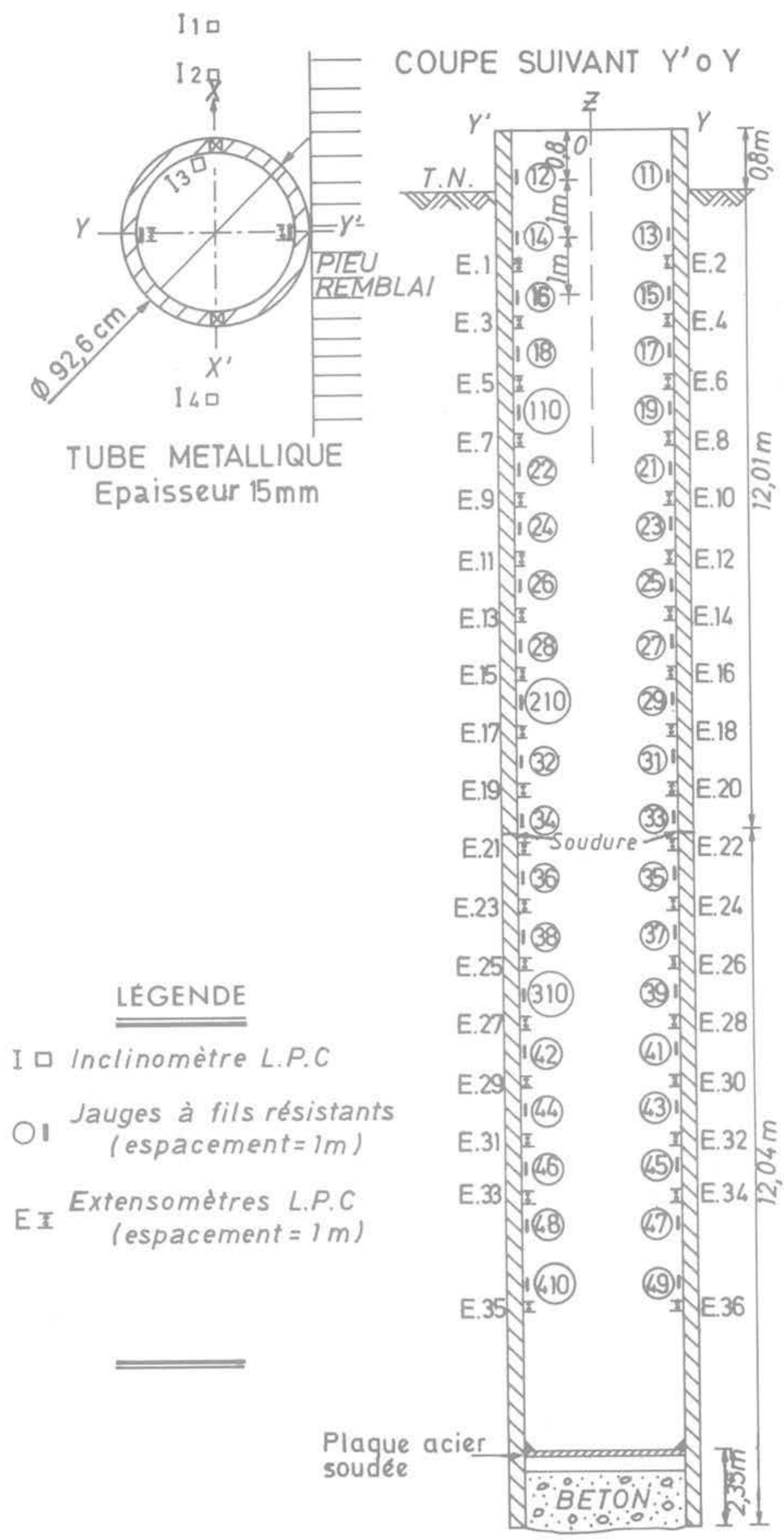

Fig. 3 Le pieu d'essai - Schéma de position des appareils 
2 L'extensomètre L. P. C. utilisé pour pallier l'inconvénient de la dérive possible des jauges avec le temps. Cet appareil (fig. 4) a été mis au point au L. C.P. C. pour la mesure des déformations sur les ouvrages d'art [4].

Comme il s'agit d'un système moins classique que les jauges, if est intéressant d'en donner une description sommaire. Sur la fibre métallique dont on veut mesurer l'élongation, sont soudées 2 pastilles portant chacune un plot cylindro-conique (fig. 5). Ces 2 plots reçoivent l'appareil de mesures. La distance entre les plots est fixée initialement à environ $200 \mathrm{~mm}$ (cette distance peut être ajustée en place par rotation de l'un des plots autour de son axe excentré).

L'appareil de mesures proprement dit est constitué par un parallélogramme déformable en métal invar dont deux des côtés sont formés de lames élastiques, les deux autres côtés ( $A$ et B, fig. 4) venant s'appliquer au moment de la mesure, sur les deux plots fixés sur la structure à tester, par l'intermédiaire de réservations coniques pratiquées dans chacun d'eux.

Le déplacement de la partie A, par rapport à la partie B, mesuré par un capteur électrique précis, donne la variation de distance entre les 2 plots soudés sur la structure.

Pour éliminer les dérives de la chaîne. de mesure et du capteur avec les variations de température, on procède par différence entre la mesure sur les plots placés sur le pieu et ceux placés sur un barreau de silice témoin, peu sensible à la température.

Par contre, on corrige les erreurs dues à la courbure du pieu par flexion et aux élongations thiermiques parasites.

\section{Mesures des déplacements horizontaux}

Les déplacements libres du sol g(z) (déplacements non influencés par le pieu) ont été mesurés à l'aide de l'inclinomètre L.P.C. modèle 1970 (sonde à pendule avec capteur angulaire descendu dans des tubes de section carrée). Sur 3 tubes préalablement mis en place, $\left(I_{1}, I_{2}, I_{4}\right.$, fig. 3$)$, seul le tube $I_{4}$ a permis de faire des mesures jusqu'à la fin des expérimentations.

En fait, on s'est aperçu que les mesures effectuées pouvaient être entachées d'erreurs importantes, dues en particulier:

- au vrillage du tube et au mauvais guidage de la sonde dans le tube (aspérités du tube; usure différentielle des roues de guidage, variation de l'effort d'application des roues contre le tubage....).

- à la sensibilité aux chocs du capteur angulaire et à la dérive de la chaîne de lecture.

- à un encastrement insuffisant du tube dans le substratum.

Les courbes obtenues étant manifestement erronées, il a fallu les corriger. Les corrections apportées sont décrites et discutées plus loin.

Pour l'essai de sollicitation horizontale, les déplacements en tête du pieu ont été mesurés par des comparateurs à partir d'une base dont la fixité était elle-même contrôlée.

Pour les autres phases de l'expérimentation, les déplacements horizontaux des têtes des inclinomètres et du pieu, ont été obtenus par triangulation à partir de trois bases fixes, la mesure étant faite à l'aide d'un théodolithe type $T_{3}$.

Les déplacements horizontaux du pieu en profondeur ont été mesurés à l'aide d'un tube inclinométrique $\left(I_{3}\right)$ solidaire de la paroi du pieu.

Mesure de la rotation en tête du pieu On a utilisé, pour cette mesure, un niveau à bulle et vis micrométrique précis solidaire de la tête du pieu. Malheureusement, la déformabilité thermique du support métallique de ce niveau a pu conduire à des erreurs non négligeables.

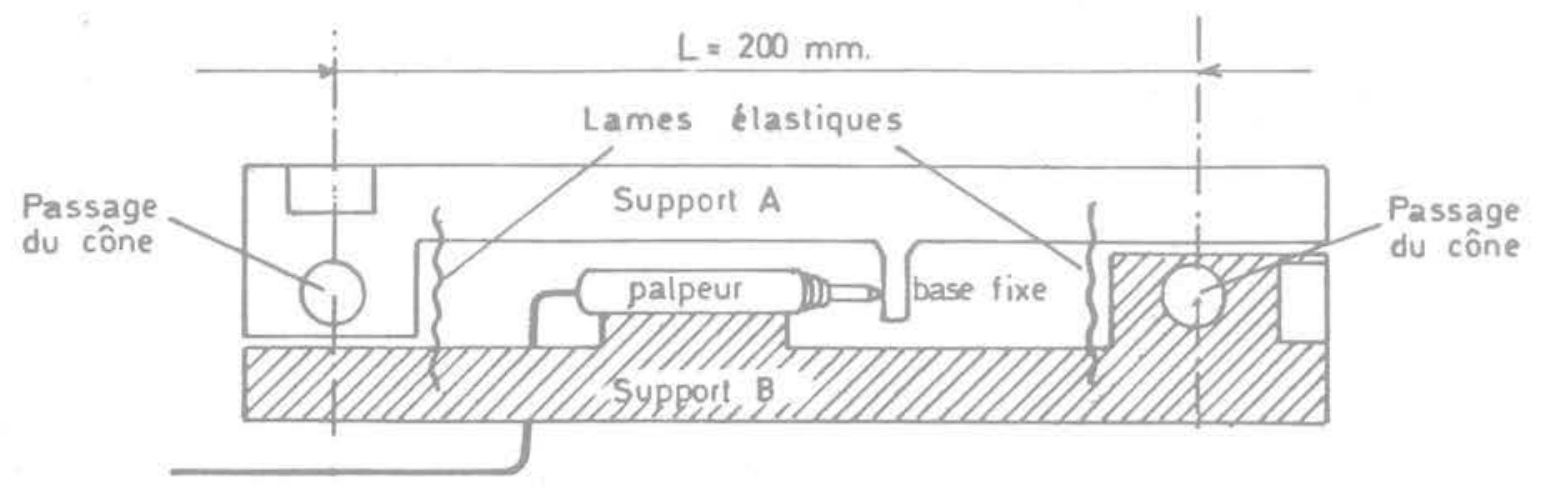

Fig. 4 Extensiomètre $\angle P C$
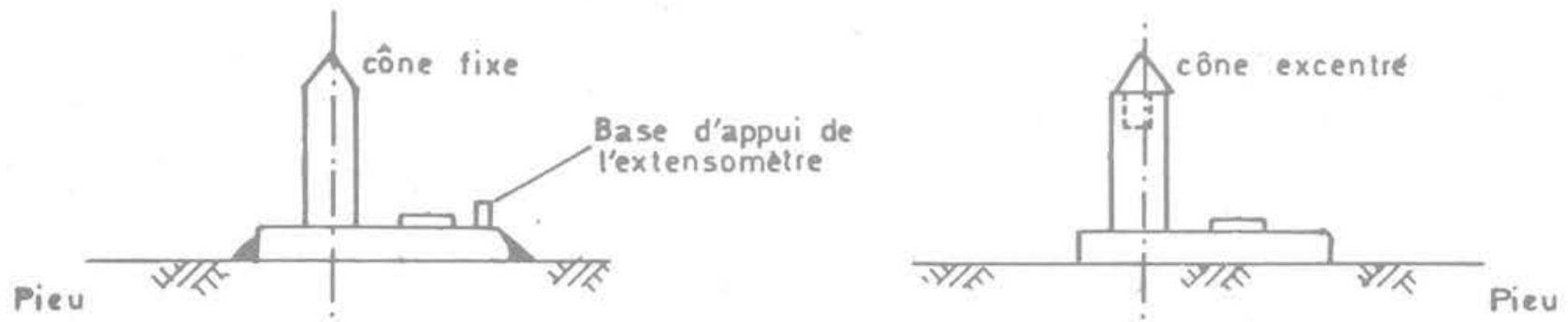

Fig. 5 Plots de mesure soudés sur le pien 


\section{Exploitation des résultats des mesures}

2.1 Principe du calcul des efforts dans le pieu à partir des mesures extensométriques

On part des mesures d'élongations $\varepsilon=\frac{\Delta \ell}{\ell}$ effectuées sur la fibre la plus tendue $\left(\varepsilon_{\tau}\right)$ et sur la fibre la plus comprimée $\left(\varepsilon_{c}\right)$ du pieu). Le moment fléchissant $M_{i}$ au niveau $i$ est donné par:

$$
M_{i}=\frac{E I}{B}\left(\varepsilon_{c_{i}}-\varepsilon_{\tau_{i}}\right)
$$

où $E$ est le module d'Young de l'acier, I le moment d'inertie transversal du pieu et B son diamètre.

Si y est le déplacement du pieu, l'équation de sa flexion s'écrit :

$$
y^{\prime \prime}=\frac{M}{E l}
$$

Par intégration jusqu'au niveau i (profondeur $z_{1}$ ) considéré et calage sur la rotation yó mesurée en tête, on a :

$$
y_{i}^{\prime}=y_{0}^{\prime}+\int_{0}^{z_{i}} \frac{M}{E l} d z
$$

Une nouvelle intégration avec calage sur le déplacement $y_{0}$ mesuré en tête donne le déplacement au niveau $i$ :

$$
y_{1}=y_{0}+\int_{0}^{z_{1}} y^{\prime} d z .
$$

Par ailleurs, l'effort tranchant est donné par :

$$
\mathrm{T}=\frac{\mathrm{dM}}{\mathrm{dz}}
$$

et la réaction du sol sur une tranche horizontale d'épaisseur dz est :

$$
\mathrm{dT}=-\mathrm{Pdz} \text {. }
$$

On peut en déduire la réaction globale unitaire p [5] (valeur moyenne rapportée à la largeur du pieu) :

$$
p=\frac{P}{B}=-\frac{1}{B} \frac{d^{2} M}{d z^{2}}(B \text { : diamètre du pieu }) \text {. }
$$

On a donc la réaction du sol au niveau i en dérivant 2 fois la courbe des moments fléchissants à ce niveau. La méthode utilisée constitue lá généralisation, à un pas de mesures variable, de la méthode de Matlock [5] [6] pour un pas de mesures constant.

Le principe consiste à accorder une valeur statistique (et non une valeur absolue) aux mesures expérimentales. On suppose que, localement, la courbe des moments est une cubique (fig. 6).

On détermine la cubique la plus probable (méthode des moindres carrés) pour un groupe de $\mathrm{N}$ points expérimentaux $(N \geqslant 5)$. Soit $\left(C_{1}\right)$ cette cubique. On considère le point central du groupe de points (niveau i) et l'on montre que l'erreur faite en prenant la cubique la plus probable est minimale en ce point.

A ce niveau, on a 2 valeurs du moment fléchissant:

- valeur expérimentale: (Mi)e.

- valeur "lissée ": $\left(\mathrm{M}_{1}\right)_{\ell}$.

La cubique dérivée localement permettra d'avoir au niveau $\mathrm{i}$ :

- l'effort tranchant: $T_{1}=\left(\frac{d M}{d z}\right)_{i}$,

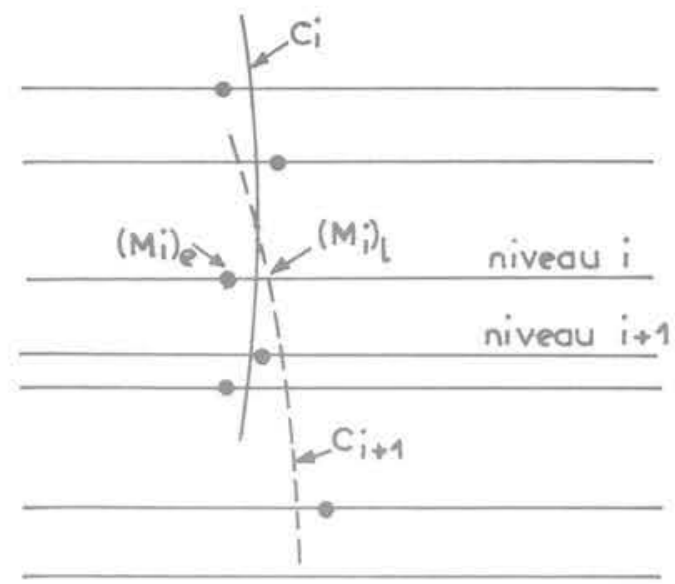

Fig. 6 Determination des cubiques locales correspondant à la courbe des moments

- la réaction du sol: $p_{1} B=P_{1}=-\left(\frac{d^{2} M}{d z^{2}}\right)_{i}$

On passe ensuite au niveau $(i+1)$ et l'on cherche la cubique $\left(C_{1+1}\right)$ la plus probable pour les $N$ points entourant le niveau $(i+1)$. On en tire de la même façon $\left(M_{i+1}\right)_{e}, T_{i+1}, P_{i+1}$, etc.

Dans le programme d'exploitation mis au point, on a retenu $\mathrm{N}=5$ et l'on tient compte de la valeur des efforts en tête $T_{0}$ et $M_{0}$ qui sont connus. C'est ainsi qu'on a obtenu pour les 4 phases d'essais considérées, les courbes donnant, en fonction de $z$, les grandeurs $M, T$, $P$ (ou p), y', y.

2.2 Comparaison des mesures d'élongation aux jauges à fils résistants et à l'extensomètre L.P.C.

Les jauges et les extensomètres sont placés, sur les mêmes génératrices verticales, mais alternativement avec un pas de $1 \mathrm{~m}$, la distance d'une jauge à l'extensomètre voisin étant donc de $0,50 \mathrm{~m}$. Par ailleurs, les élongations sont mesurées sur des longueurs différentes : $2 \mathrm{~cm}$ pour les jauges et $20 \mathrm{~cm}$ pour les extensomètres. Pour faire des comparaisons à un niveau, on doit faire une interpolation linéaire sur les mesures voisines sur l'un des 2 types d'appareil. II faut donc se placer en des zones où les réponses varient à peu près linéairement avec la profondeur. On a choisi deux niveaux où ce critère était à peu près respecté soit à $-3,65 \mathrm{~m}$ et à $-12,15 \mathrm{~m}$ par rapport au terrain naturel.

Les comparaisons sont données sur les figures 7 et 8 .

Pour le $1^{\text {er }}$ niveau (fig. 7), on compare, pour la fibre tendue et pour la fibre comprimée, l'élongation donnée par un extensomètre à la moyenne des réponses des jauges voisines. On fait l'inverse pour le $2^{\circ}$ niveau (fig. 8). On compare également les différences des réponses des deux types d'appareil, entre la fibre tendue et la fibre comprimée, le moment fléchissant étant proportionnel à ces différences.

De cette comparaison qui avait pour but de vérifier une éventuelle dérive des jauges pendant la durée des mesures (6 mois environ), la référence étant la mesure à l'extensomètre L.P.C., on peut tirer globalement les conclusions suivantes:

1) Sur l'intervalle de temps considéré, il n'apparaît pas de dérive des jauges. 


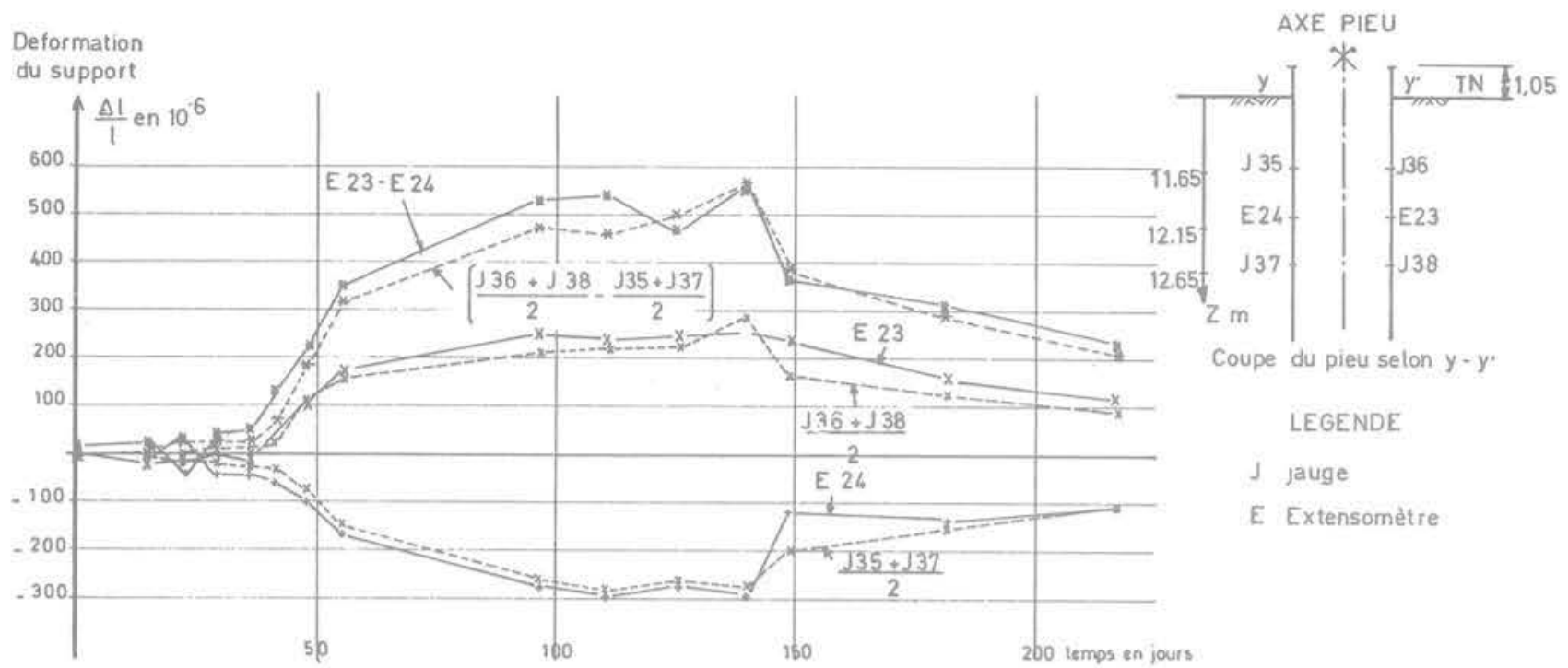

Fig. 7 Comparaison entre les jauges et les extensomètres. Niveau : $12,15 \mathrm{~m} / \mathrm{TN}$

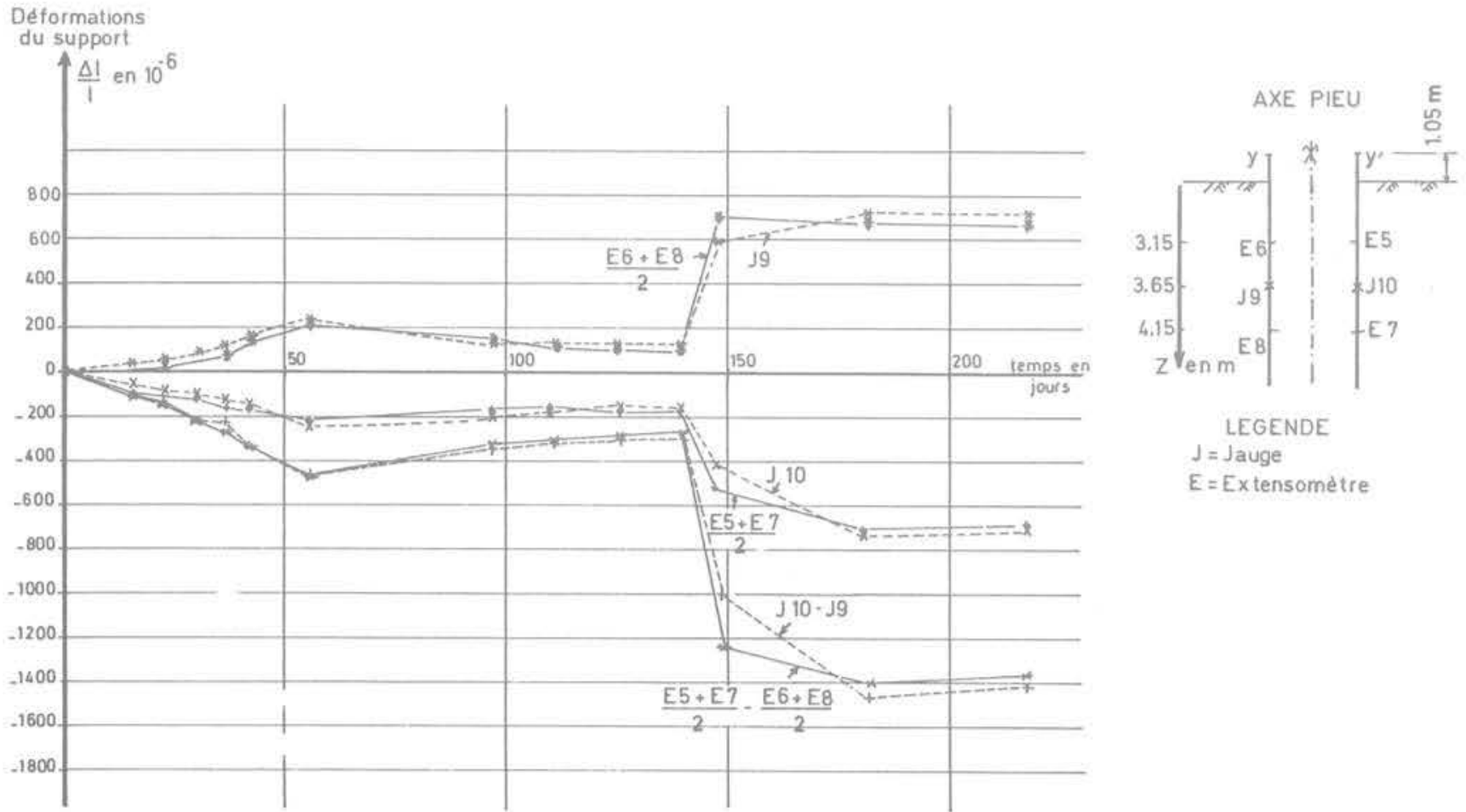

Fig. 8 Comparaison entre les jauges et les extensomètres. Niveau : $3,65 \mathrm{~m} / \mathrm{TN}$ 
2) On note cependant un écart qui n'est pas toujours dans le même sens entre la réponse des jauges et celle des extensomètres: elle peut s'expliquer par la différence de longueur des bases de mesures (rapport de 1 à 10). En général, cet écart reste inférieur à $50 \cdot 10^{-6}$. Toutefois, l'écart peut se cumuler sur les fibres tendues et comprimées et conduire à un écart sur le moment fléchissant de l'ordre de $100 \mathrm{kN} \times \mathrm{m}$ et ceci, quelle que soit la valeur de ce moment. L'écart relatif possible, important pour les faibles valeurs du moment, décroît donc lorsque le moment fléchissant augmente et se situe à peu près à $\pm 7 \%$ pour les moments maximaux mesurés (de l'ordre de $1500 \mathrm{kN} \times$ $\mathrm{m})$.

\subsection{Mesures inclinométriques}

Pour les raisons indiquées plus haut, les erreurs sur les mesures inclinométriques ont été très importantes. Les déformées retenues pour les comparaisons avec les calculs, ont été obtenues:

1) pour le pieu par double intégration des courbes des moments et calage sur la rotation et le déplacement mesurés en tête, soit $y(z)$.

2) pour le sol «libren, (déplacement du sol, en l'absence du pieu, ou loin du pieu, soit $g(z)$ ) par correction des courbes inclinométriques brutes par la méthode expliquée ci-après :

Les déplacements en tête (mesures topométriques) sont supposés corrects. Par ailleurs, on détermine sur les courbes donnant la pression moyenne sur le pieu (obtenues par double dérivation des courbes de moments) les points de pression nulle. Pour les phases de chargement du pieu par le remblai, il y a deux points de pression nulle (fig. 9) correspondant à $y=g$. Comme on connaît le déplacement y du pieu (intégration de Ely"), on en tire g en ces points. On déduit la déformée du sol libre corrigée, de la courbe inclinométrique brute par une affinité dont le rapport est déterminé à partir de la moyenne des rapports $g_{\text {réel }} / g_{\text {brut }}$ aux points de pression nulle. Cette méthode d'interprétation, en soi fort discutable, aboutit à des déformées dont l'allure est vraisemblable et pourra être comparée à celle déduite de la méthode de prévision des déformées du sol libre présentée plus loin.

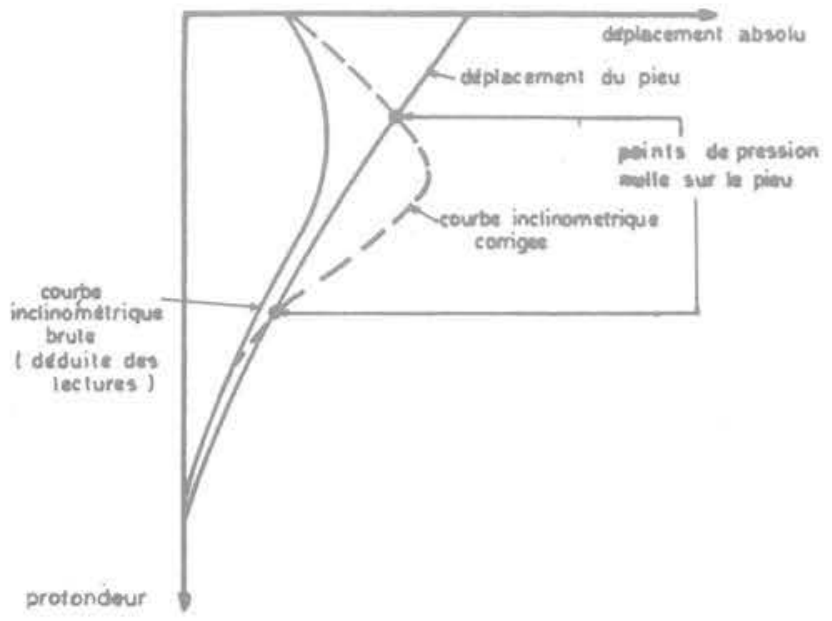

3 Efforts et déplacements : comparaison entre les résultats des mesures et les prévisions du calcul

\subsection{Principe de la méthode de calcul}

On rappelle succintement le principe de cette méthode qui a déjà fait l'objet de plusieurs publications [1], [2], [7], [8], [9]. La notion de module de réaction utilisée pour l'étude théorique des pieux soumis à des efforts de flexion en tête [5], est étendue au cas des pieux subissant l'action du déplacement latéral du sol de fondation sous un remblai, en prenant en compte la différence $\Delta y=y-g$ entre le déplacement du pieu y et le déplacement libre du sol g au lieu du déplacement y. L'équation d'équilibre des pressions s'écrit :

$$
\text { EI } \frac{d^{4} y}{d z^{4}}+E_{s}(z, \Delta y) \cdot \Delta y=0 \text {. }
$$

Dans cette équation, $E_{8}$, module horizontal du sol, est donné par les pentes des sécantes de la courbe de réaction à la cote considérée (fig. 10). (Sur la figure, p représente la pression moyenne sur le pieu et B le diamètre du pieu).

Le problème est résolu numériquement (programme PILATE des L.P.C.) à partir d'une solution analytique pour chaque couche de sol dans laquelle on peut considérer la loi de réaction comme unique et où l'on peut exprimer la fonction $\mathrm{g}(\mathrm{z})$ par un polynôme de degré inférieur ou égal à 3 . La continuité aux intercouches ainsi que les conditions aux limites en tête et en pointe permettent de calculer les constantes d'intégration. La non-linéarité de la loi de réaction nécessite un processus itératif.

La prévision des déplacements libres du sol $g(z)$ et le choix des courbes de réaction sont les deux problèmes principaux qui se posent pour l'application de la méthode. Ils sont examinés ci-après.

Notons que dans le cas du pieu sollicité horizontalement en tête, sans chargement du sol par un remblai, la formulation est la même, avec $g(z) \equiv 0$ donc $\Delta y \equiv y$.

\subsection{Hypothèses de calculs pour le remblai de Provins}

Le schéma général de calcul est donné sur la figure 1.

Déformée libre du sol $(\mathrm{g}(\mathrm{z}))$

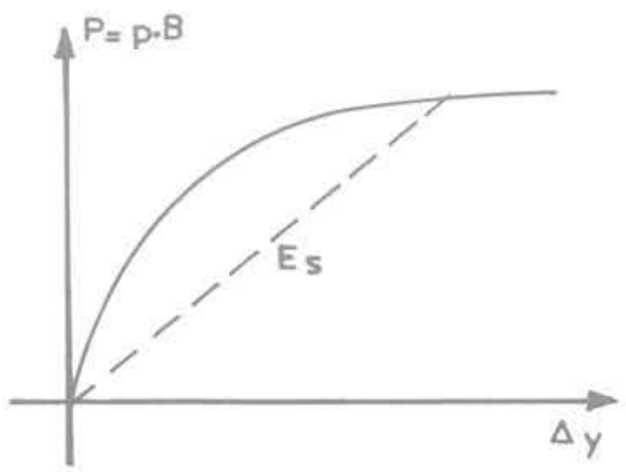

Fig. 10 Courbe de réaction 
Elle est déterminée par la méthode récemment proposée [2], [10], [11]. Rappelons brièvement cette méthode pour laquelle les notations sont données (fig. 11).

L'équation de la déformée libre en coordonnées réduites :

$$
G=g / g_{\max }, \quad Z=z / D
$$

est donnée par:

$$
G=1,83 Z^{3}-4,69 Z^{2}+2,13 Z+0,73
$$

Connaissant $\mathrm{g}_{\max }$, on peut déterminer $\mathrm{g}(\mathrm{z})$. Au temps $\mathrm{t}$, $g_{\max }$ est donné par:

$$
g_{\max , t}=g_{\max , 0}+0,16\left(s_{1}-s_{0}\right)
$$

$g_{\max , 0}$ est le déplacement latéral maximal en fin de construction, $s_{0}$ et $s_{t}$, les tassements sous l'axe du remblai respectivement en fin de construction et au temps t.

On a $g_{\max , 0}=\lambda \cdot \mathrm{D}, \lambda$ étant déterminé à partir de l'abaque de la figure 12 , où :

$-f=\frac{(\pi+2) \bar{c}_{u}}{\gamma_{r} H}$ est un paramètre de charge, et $-m=\frac{1+\sin ^{2} \beta}{\sin \beta^{\prime}}$, un paramètre géométrique.

Notons que $\bar{c}_{u}$ est la cohésion moyenne dans la couche molle.

\section{Application au remblai de Provins}

Les calculs sont effectués pour les phases 1,2 et 3 définies plus haut.

phase 1: $18 \cdot 07 \cdot 74, \mathrm{t}=48$ jours (fin de construction à $3,80 \mathrm{~m})$;

phase 2: $30 \cdot 07 \cdot 74, t=60$ jours (fin de construction à $6,80 \mathrm{~m})$;

phase 3: $29 \cdot 10 \cdot 74, t=151$ jours (remblai toujours à $6,80 \mathrm{~m}$ après consolidation partielle du sol de fondation).
La moyenne calculée sur 50 mesures de cohésion au scissomètre entre 0 et $14,50 \mathrm{~m}$ donne :

$\overline{\mathrm{c}}_{\mathrm{u}}=48 \mathrm{kPa}$. (On suppose qu'il n'y a aucune variation de $\bar{c}_{u}$ entre 0 et 60 jours.)

On a $\gamma_{r}=20 \mathrm{kN} / \mathrm{m}^{3}, D=14,50 \mathrm{~m}, \mathrm{~m}=2,68$.

Pour $\mathrm{t}=48$ jours

$$
\begin{gathered}
f=3,25 \text { conduit à } \lambda=0,33 \\
\text { d'où } g_{\max , 0}=4,8 \mathrm{~cm}
\end{gathered}
$$

Pour $\mathrm{t}=60$ jours

$$
\begin{gathered}
f=1,81 \text { conduit à } \lambda=1,13 \\
\text { d'où } g_{\max , 0}=16,4 \mathrm{~cm} .
\end{gathered}
$$

Pour $t=151$ jours $(H=6,80 \mathrm{~m})$

$$
g_{\max , 151 j}=g_{\max , 60 j}+0,16\left(s_{151 j}-s_{60 j}\right) .
$$

Les mesures de tassement ont donné :

$$
\begin{aligned}
\mathrm{S}_{151 \mathrm{j}} & =172 \mathrm{~cm} \\
\mathrm{~S}_{601} & =90 \mathrm{~cm}
\end{aligned}
$$

donc $g_{\max , 151 j}=16,4+0,16(172-90)=29,5 \mathrm{~cm}$.

L'équation de $g(z)$ est alors :

Phase 1 (48 jours) :

$$
g_{1}=0,00003 z^{3}-0,00107 z^{2}+0,00706 z+0,0350
$$

Phase 2 (60 jours) :

$$
g_{2}=0,00010 z^{3}-0,00366 z^{2}+0,02412 z+0,1197
$$

\begin{tabular}{|c|c|c|c|c|c|c|c|c|c|}
\hline Couches & $\begin{array}{c}\mathrm{P}_{1} \\
\mathrm{kPa}\end{array}$ & $\begin{array}{r}\mathrm{E}_{\mathrm{M}} \\
\mathrm{kPa}\end{array}$ & $\alpha$ & $\begin{array}{c}\mathrm{E}_{\mathrm{s}} \\
\mathrm{kPa}\end{array}$ & $\begin{array}{l}p_{\mathrm{f}} \cdot \mathrm{B} \\
\mathrm{kN} / \mathrm{m}\end{array}$ & $\begin{array}{c}(y-g)_{t} \\
\text { en } \\
10^{-3} \mathrm{~m}\end{array}$ & $\begin{array}{l}p_{1} \cdot B \\
k N / m\end{array}$ & $\begin{array}{c}(y-g)_{1} \\
\text { en } \\
10^{-3} \mathrm{~m}\end{array}$ & 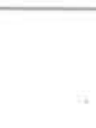 \\
\hline $0-4 m(1)$ & 60 & 160 & 1100 & 0,5 & 2937 & 55,6 & 18,9 & 148,2 & 82,0 \\
\hline-4 à $-14,50(2)$ & 120 & 250 & 1070 & 1 & 1416 & 111,1 & 78,5 & 231,5 & 248,5 \\
\hline $\begin{array}{c}-14,50 \text { à } \\
-17,5\end{array}$ & 660 & 1190 & 3600 & 0,25 & 14608 & 611,2 & 41,8 & 1101,9 & 109,0 \\
\hline $\begin{array}{c}-17,50 \mathrm{a} \\
-18,50\end{array}$ & 360 & 650 & 900 & 0,66 & 1877 & 333,4 & 177,0 & 601,9 & 463,7 \\
\hline $\begin{array}{c}-18,50 \text { à } \\
-20,50\end{array}$ & 360 & 625 & 1900 & 0,66 & 3963 & 333,4 & 84,1 & 578,7 & 207,9 \\
\hline $\begin{array}{c}-20,50 \mathrm{a} \\
-21,50\end{array}$ & 320 & 720 & 3100 & 0,66 & 6467 & 296,3 & 45,8 & 666,7 & 160,4 \\
\hline $\begin{array}{c}-21,50 \mathrm{à} \\
-23,00\end{array}$ & 675 & 1250 & 6700 & 0,66 & 13977 & 625,0 & 44,7 & 1157,5 & 120,9 \\
\hline
\end{tabular}

Phase 3 ( 151 jours) :

$$
g_{3}=0,00018 z^{3}-0,00658 z^{2}+0,04340 z+0,2153
$$

Les courbes $\mathrm{g}(\mathrm{z})$ sont données sur la figure 13 où l'on a reporté également les déformées libres du sol déduites des mesures inclinométriques corrigées effectuées aux mêmes dates.

Choix des lois de réaction $\mathrm{P}=\mathrm{f}(\Delta \mathrm{y})$.

Trois types de lois de réaction ont été utilisées. Elles seront dénommées par la suite loi A, Ioi B, loi C.

Tableau $n^{\circ} 3$

Données des lois de réaction A (Ménard) pour les différentes couches de sol.

(1) divisée en 3 sous-couches $0-1 \mathrm{~m}, 1-2,50 \mathrm{~m}, 2,50-4 \mathrm{~m}$, avec la même loi de réaction.

(2) divisée en 5 sous-couches $4-6-8-10-12-14,50 \mathrm{~m}$ avec la même loi de réaction. 


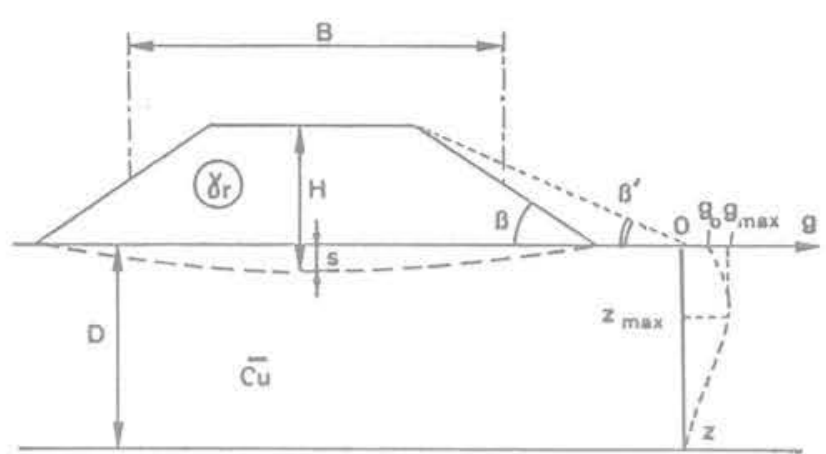

Fig. 11 Définition des paramètres utilisés pour la prévision de la déformée du sol libre
Loi $A$. La courbe de réaction est tracée à partir du module du sol $\mathrm{E}_{\mathrm{s}}$ déduit d'un essai pressiométrique normal (essai Ménard).

Les résultats de l'essai $P_{1}$ utilisé (pression limite $p_{1}$, module pressiométrique standard $E_{M}$ ) sont donnés sur la figure 2. On en tire pour chaque couche la loi de réaction à 3 pentes préconisée par Ménard (fig. 14).

On a découpé le sol en couches à peu près homogènes et les coordonnées des points de la courbe de réaction titrés des valeurs moyennes de $p_{t}, p_{1}, E_{s}$ sont données dans le tableau $n^{\circ} 3, E_{5}$ étant calculé par la formule

$$
E_{s}=E_{M} \frac{18}{4\left(2,65 \frac{B}{B_{0}}\right)^{\alpha} \frac{B_{0}}{B}+3 \alpha}
$$

dans laquelle $B_{0}=0,60 \mathrm{~m}$ et $\alpha$ est le coefficient caractérisant le type de sol.

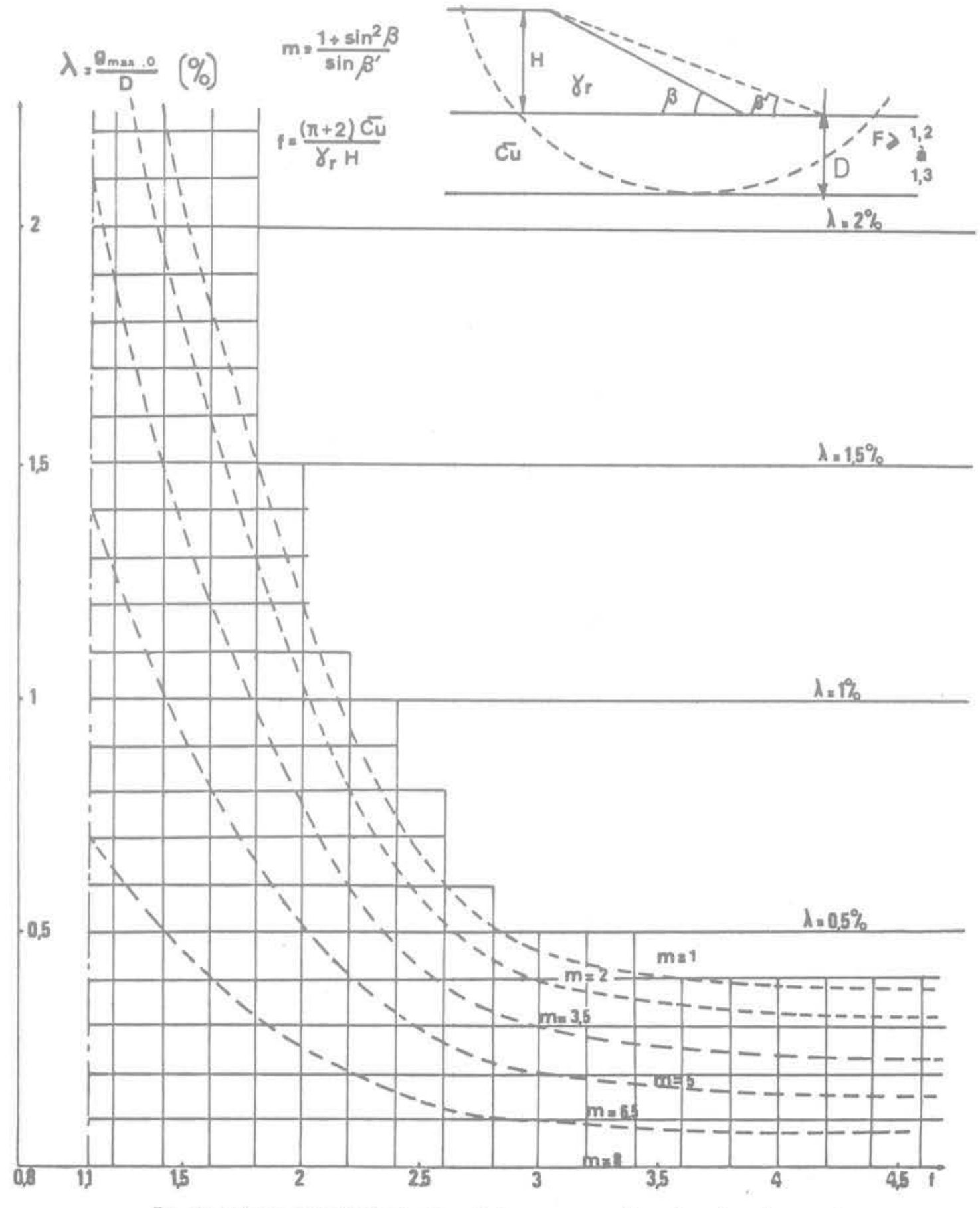

Fig. 12 Abaque de détermination de $\lambda=g_{\max , 0} / d$ en fonction de $m$ et $f$ 
Loi B. La courbe de réaction est tracée à partir du module du sol $\mathrm{E}_{\mathrm{a}}$ déduit d'un essai au pressiomètre autoforeur.

L'essai au pressiomètre autoforeur (P.A.F.) (essai de référence: déformation volumique $\Delta \mathrm{V} / \mathrm{V}_{0}$ de $2 \%$ par minute) effectué après l'expérience, à proximité du pieu, a donné les résultats fournis au tableau $n^{\circ} 4$ dans lequel $\beta$ est le coefficient d'identification des sols, $p_{2}, p_{5}$ et $p_{20}$ les pressions à $0,2,5$ et $20 \%$ de déformation volumique, $\mathrm{G}_{\mathrm{P}_{2}}$ et $\mathrm{G}_{\mathrm{p}_{5}}$ les modules de cisaillement sécants à 2 et $5 \%$ de déformation.

Par analogie avec la méthode proposée par Ménard, et à partir de la formule permettant de déterminer les tassements en se basant sur les résultats de l'essai P. A. F., on tire la formule donnant le module du sol :

$$
E_{s}=\frac{10}{2 \frac{B_{0}}{B} \frac{1}{G_{p_{2}}}\left(2,65 \frac{B}{B_{0}}\right)^{1-\beta}+\frac{1,5}{G_{p_{5}}}}
$$

On retient comme loi de réaction dans chaque couche, une courbe à 3 pentes du type de celle qui est donnée sur la figure 15.

Les coordonnées des points de cette courbe, pour chaque couche à peu près homogène retenue, et tirées des caractéristiques moyennes de cette couche, sont données dans le tableau $n^{\circ} 5$. Les couches épaisses ont été divisées en plusieurs sous-couches de même courbe de réaction, pour le calcul. Dans ce tableau, on a :

$$
p_{20}^{*}=p_{20}-p_{0}
$$

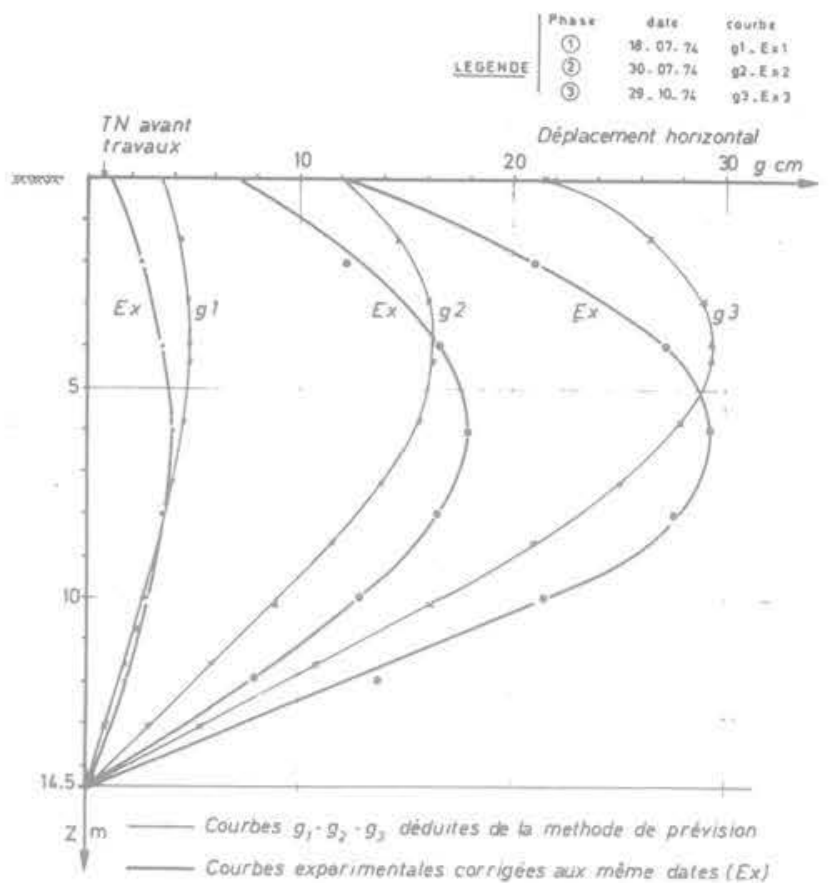

Fig. 13 Déplacement horizontal du sol

\begin{tabular}{|c|c|c|c|c|c|c|c|c|}
\hline $\begin{array}{c}\text { profondeur \% } \\
\text { au T.N. }\end{array}$ & $\beta=\frac{p_{20}-p_{5}}{p_{20}-p_{0}}$ & $P_{20}$ & \multicolumn{2}{|c|}{$P_{5} \prod_{k P a} P_{2}$} & $\mathrm{p}_{\mathrm{o}}$ & \multicolumn{2}{|c|}{$\mathrm{kPa}$} & $\begin{array}{c}\mathrm{E}_{\mathrm{s}} \\
\mathrm{kPa}\end{array}$ \\
\hline $0,50 \mathrm{~m}$ & 0,51 & 200 & 104 & 64 & 11 & 1870 & 2660 & 5638 \\
\hline 1,50 & 0,40 & 158 & 103 & 74 & 20 & 1670 & 2710 & 4971 \\
\hline 2,50 & 0,35 & 181 & 128 & 98 & 30 & 1950 & 3380 & 5790 \\
\hline 3,50 & 0,48 & 183 & 120 & 90 & 53 & 1350 & 1900 & 3953 \\
\hline 4,50 & 0,47 & 171 & 117 & 87 & 56 & 1210 & 1530 & 3304 \\
\hline 5,50 & 0,44 & 196 & 140 & 106 & 69 & 1430 & 1900 & 3922 \\
\hline 6,50 & 0,45 & 183 & 138 & 113 & 82 & 1110 & 1560 & 3171 \\
\hline 7,50 & 0,43 & 207 & 156 & 125 & 89 & 1330 & 1800 & 3657 \\
\hline 8,50 & 0,42 & 216 & 166 & 134 & 96 & 1390 & 1900 & 3812 \\
\hline 9,50 & 0,45 & 237 & 194 & 173 & 142 & 1030 & 1530 & 3036 \\
\hline 10,50 & 0,39 & 216 & 176 & 152 & 113 & 1250 & 1950 & 3611 \\
\hline 11,50 & 0,40 & 260 & 206 & 170 & 125 & 1620 & 2260 & 4423 \\
\hline 12,50 & 0,45 & 286 & 220 & 182 & 138 & 1630 & 2180 & 4525 \\
\hline 13,50 & 0,28 & 307 & 261 & 228 & 143 & 2350 & 4240 & 6753 \\
\hline
\end{tabular}

Tableau 4 Essai au PAF 


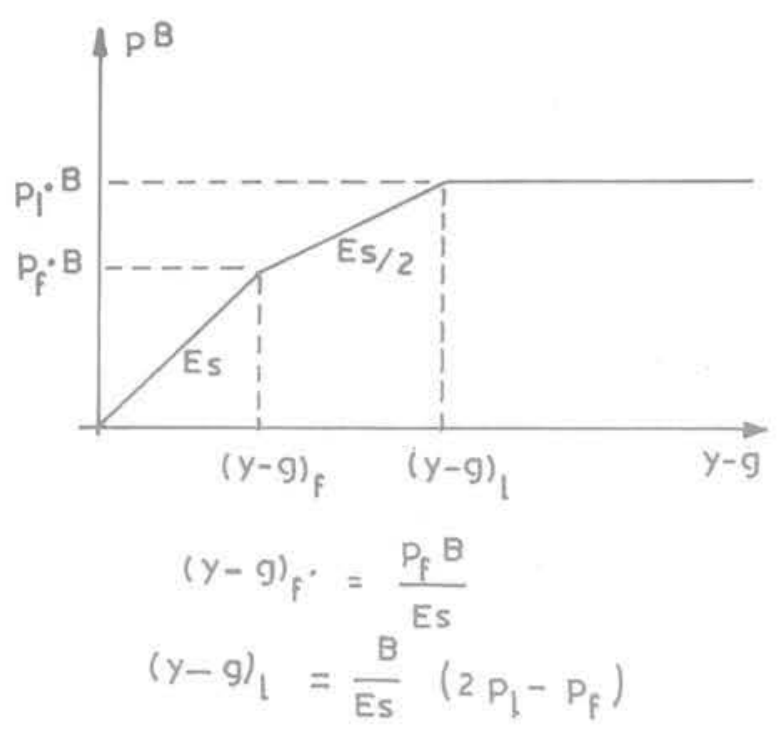

Fig. 14 Loi de réaction d'après Ménard : Ioi A

L'essai au P.A. F. n'ayant pas été poursuivi au-delà de $13,50 \mathrm{~m}$, pour les couches de graviers puis de craie entre $14,50 \mathrm{~m}$ et $23 \mathrm{~m}$, on a retenu les courbes de réaction données au tableau $n^{\circ} 3$ et tirées de l'essai au pressiomètre normal.

Loi C. La courbe d'expansion au P.A.F. est utilisée comme loi de réaction.

On a utilisé le même essai que pour la loi B.

Conformément aux plus récentes recommandations concernant les applications de l'autoforage [12], on a pris comme courbe de réaction:

- pour l'essai de sollicitation horizontale en tête (chargement rapide), la courbe $P, \Delta y$ déduite de la courbe d'expansion au P.A.F. $\left(p, \frac{\Delta V}{V_{0}}\right)$ par le changement de variable :

$$
\begin{array}{r}
P=\left(p-p_{0}\right) B \\
\Delta y=\frac{B}{4} \frac{\Delta V}{V_{0}}
\end{array}
$$

- pour le chargement par le remblai (chargement permanent), une loi semblable, mais pour laquelle, par rapport à la loi $\mathrm{C}_{1}$, les déplacements sont doublés pour une pression donnée (Loi $\mathrm{C}_{2}$ ) (fig. 16).

Pour les graviers et la craie $(14,50 \mathrm{~m}$ à $23 \mathrm{~m})$, on a ici encore utilisé les lois de réaction $\mathrm{A}$ tirées de l'essai "Ménard" (tableau $n^{\circ} 3$ ).

Tableau $n^{\circ} 5$

Données des lois de réaction B (issues de l'essai au P.A.F.) pour les différentes couches de sol

\begin{tabular}{|c|c|c|c|c|c|}
\hline Couches de sol & $\begin{array}{c}\overline{\mathrm{E}}_{\mathrm{s}} \\
\mathrm{kPa}\end{array}$ & $\begin{array}{c}\overline{\mathrm{p}_{20}^{*} \cdot \mathrm{B}} \\
\mathrm{kN} / \mathrm{m}\end{array}$ & $\begin{array}{c}\overline{(\mathrm{y}-\mathrm{g})_{2}} \\
10^{-3} \mathrm{~m}\end{array}$ & $\begin{array}{c}\frac{1}{2 \mathrm{p}_{20}^{*} \cdot \mathrm{B}} \\
\mathrm{kN} / \mathrm{m}\end{array}$ & $\begin{array}{c}\overline{(\mathrm{y}-\mathrm{g})_{1}} \\
10^{-3} \mathrm{~m}\end{array}$ \\
\hline $\begin{array}{c}\text { O à 3 m } \\
\text { (avec 2 sous-couches) }\end{array}$ & 5466 & 147,54 & 40,50 & 73,77 & 13,50 \\
\hline $\begin{array}{c}\text { 3 à 11 } \mathrm{m} \\
\text { (avec 4 sous-couches) }\end{array}$ & 3558 & 105,22 & 44,57 & 52,61 & 14,79 \\
\hline 11 à 13 m & 4474 & 131,03 & 43,92 & 65,52 & 14,64 \\
\hline 13 à 14,50 m & 6753 & 151,86 & 33,72 & 75,93 & 11,24 \\
\hline
\end{tabular}




\section{Hypothèses particulières}

Conditions en tête et en pointe

En tête $(z=0)$, le pieu est libre, avec un moment $M_{0}$ nul et un effort tranchant $T_{0}$ nul dans le cas du chargement par le remblai, et $T_{0}$ égal à $120 \mathrm{kN}$ et $\mathrm{M}_{0}=24 \mathrm{kN} \cdot \mathrm{m}$ pour l'essai de sollicitation horizontale avant mise en place du remblai.

En pointe $(z=23 \mathrm{~m})$, les conditions ont été figurées par une longueur fictive supplémentaire du pieu de $0,3 \mathrm{~B}=0,30 \mathrm{~m}$ dans la dernière couche, le pieu ainsi allongé étant considéré comme libre en pointe avec $M_{p}=T_{p}=0$.

Diminution de la réaction pour les couches proches de la surface

Si $z_{c}$ représente la profondeur critique pour le pieu, les lois de réaction pour $z<z_{c}$ sont modifiées de la façon suivante : pour un déplacement $\Delta y$ donné, la pression est affectée d'un coefficient minorateur égal à $\left(1+z / z_{c}\right) / 2$.

On a pris ici $\mathrm{z}_{\mathrm{c}}=2 \mathrm{~B} \approx 2 \mathrm{~m}$.

3.3 Comparaison entre les résultats des mesures et les prévisions du calcul

Pieu libre avec efforts nuls en tête $\left(T_{0}=M_{0}=0\right)$ Chargement par le remblai (Phases 1, 2, 3)

Examen des courbes de Moments fléchissants et déplacements

Les figures 17 à 22 donnent, pour chacune des phases, 4 courbes et ceci pour les moments et déplacements du pieu.

(M) : courbe déduite des mesures.

(A), $(B),\left(C_{2}\right)$ : courbes calculéés avec respectivement :
(A) : loi de réaction $A$
(B) : loi de réaction $\mathrm{B}$
$\left(C_{2}\right)$ : loi de réaction $C_{2}$.

L'examen de ces courbes conduit aux constatations globales suiantes :

a) Les valeurs maximales des moments se situent sensiblement aux mêmes cotes pour les calculs et pour les mesures: milieu de la couche molle et niveau du substratum. Qualitativement, les courbes de moments et de déplacements observées sont tout à fait semblables aux courbes données par les calculs. b) Les moments calculés sont trop faibles en tête (surtout aux faibles charges) et toujours trop forts en pied (tableau $n^{\circ} 6$ ).

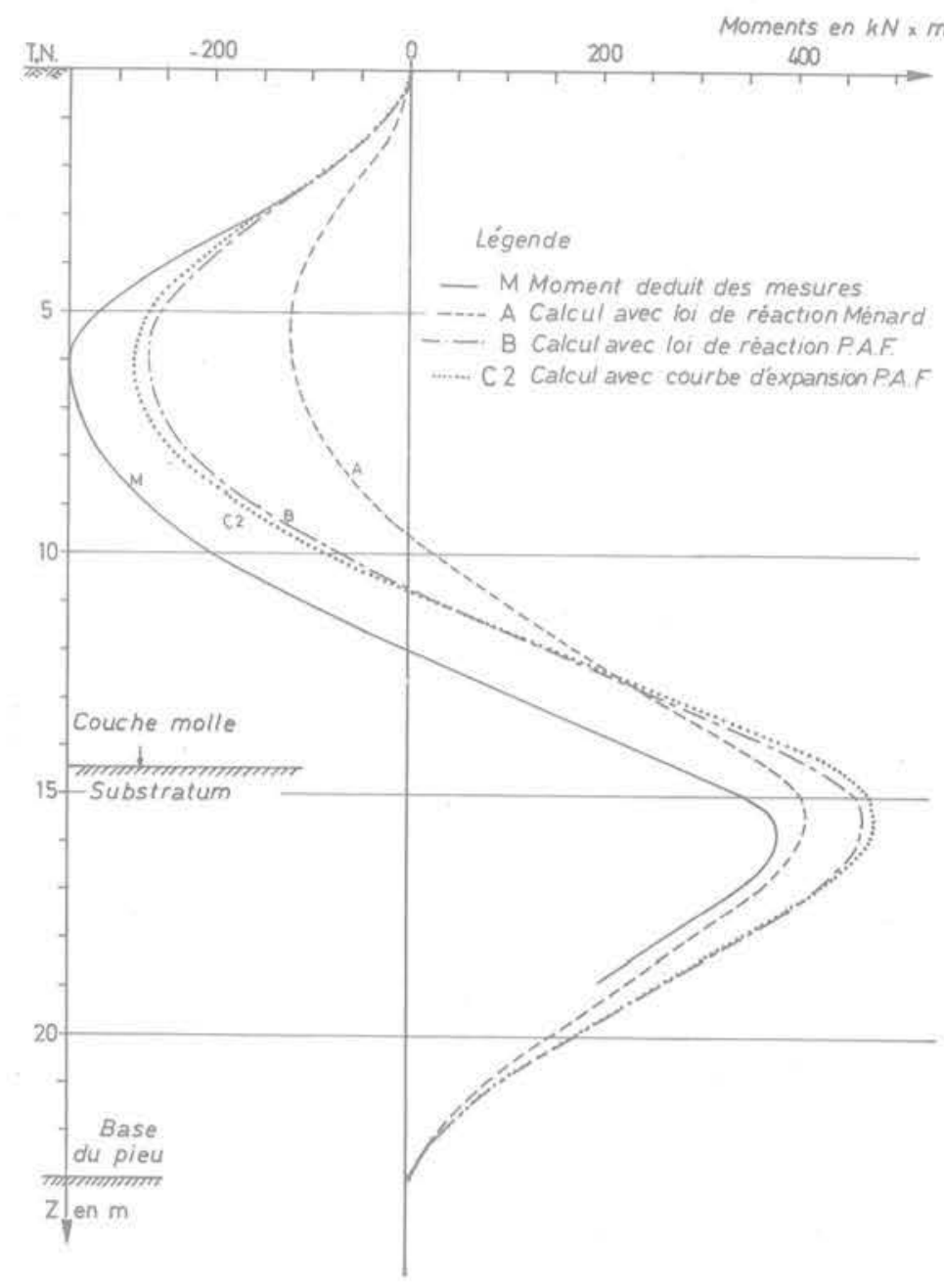

Fig. 17 Moments dans le pieu au 18.07.74, phase 1

Tableau 6

Rapport $\frac{\text { Moment maximal calculé }}{\text { Mment maximal mesuré }}$

\begin{tabular}{|c|c|c|c|c|}
\hline & & $\begin{array}{l}\text { Calcul } \\
\text { loi } \mathrm{A}^{*}\end{array}$ & $\begin{array}{l}\text { Calcul } \\
\text { loi } \mathrm{B}^{*}\end{array}$ & $\begin{array}{l}\text { Calcul } \\
\text { loi } \mathrm{C}_{2}\end{array}$ \\
\hline \multirow{3}{*}{$\begin{array}{c}\text { Maximum } \\
\text { couche } \\
\text { molle }\end{array}$} & Phase 1 & 0,35 & 0,76 & 0,81 \\
\hline & Phase 2 & 0,46 & 0,93 & 0,76 \\
\hline & Phase 3 & 0,93 & 1,35 & 1,10 \\
\hline \multirow{3}{*}{$\begin{array}{c}\text { Moment } \\
\text { d'encastrement }\end{array}$} & Phase 1 & 1,08 & 1,23 & 1,25 \\
\hline & Phase 2 & 1,30 & 1,46 & 1,43 \\
\hline & Phase 3 & 1,52 & 1,63 & 1,58 \\
\hline
\end{tabular}




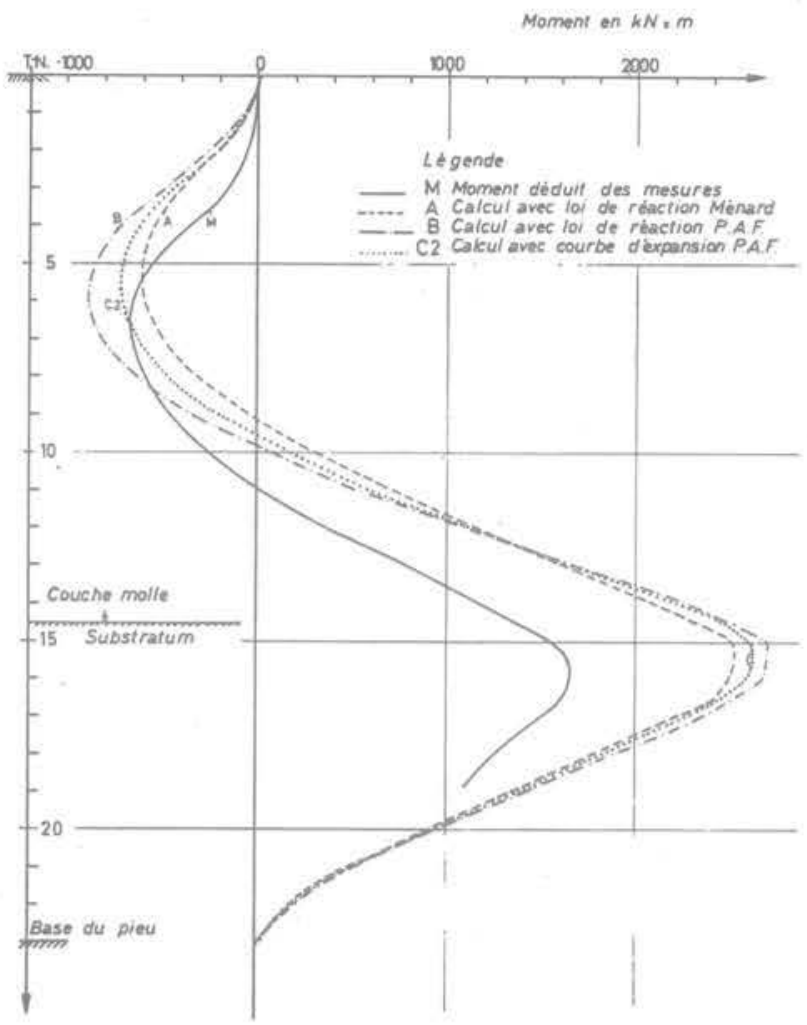

Fig. 18 Moments dans le pieu au 30.07.74, phase 2

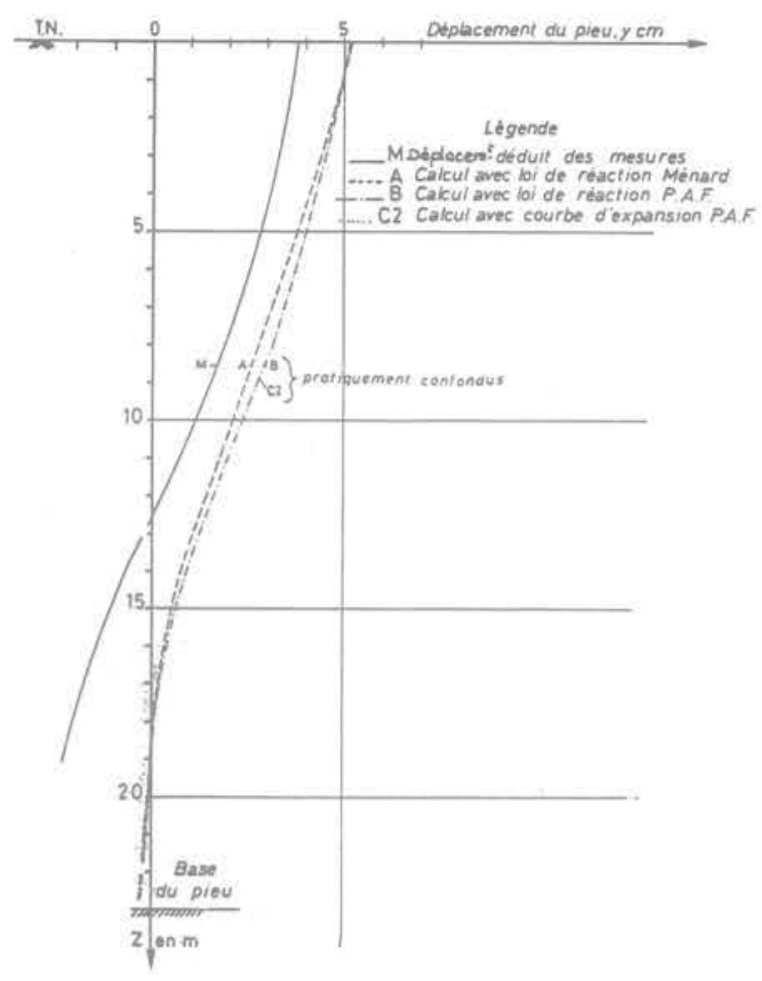

Fig. 20 Déplacements du pieu au 18.7.74

$$
\begin{gathered}
M \text { courbe déduite des mesures } \\
A \text { calcul avec la loi de réaction Ménard } \\
B \text { calcul avec la loi de réaction PAF } \\
C_{1} \text { calcul avec courbe d'expansion PAF } \\
C_{2} \text { (déplacements non doublés) } \\
\text { (déplacements avec courbe d'expansion PAF }
\end{gathered}
$$

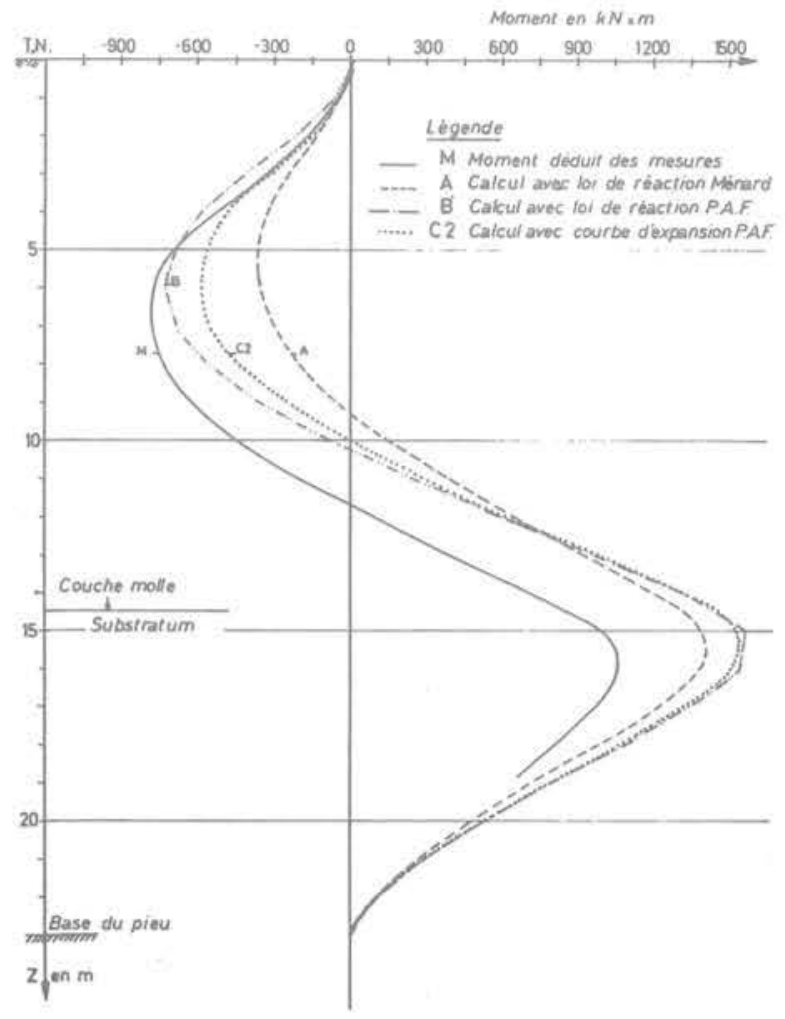

Fig. 19 Moments dans le pieu au 29.10.74, phase 3

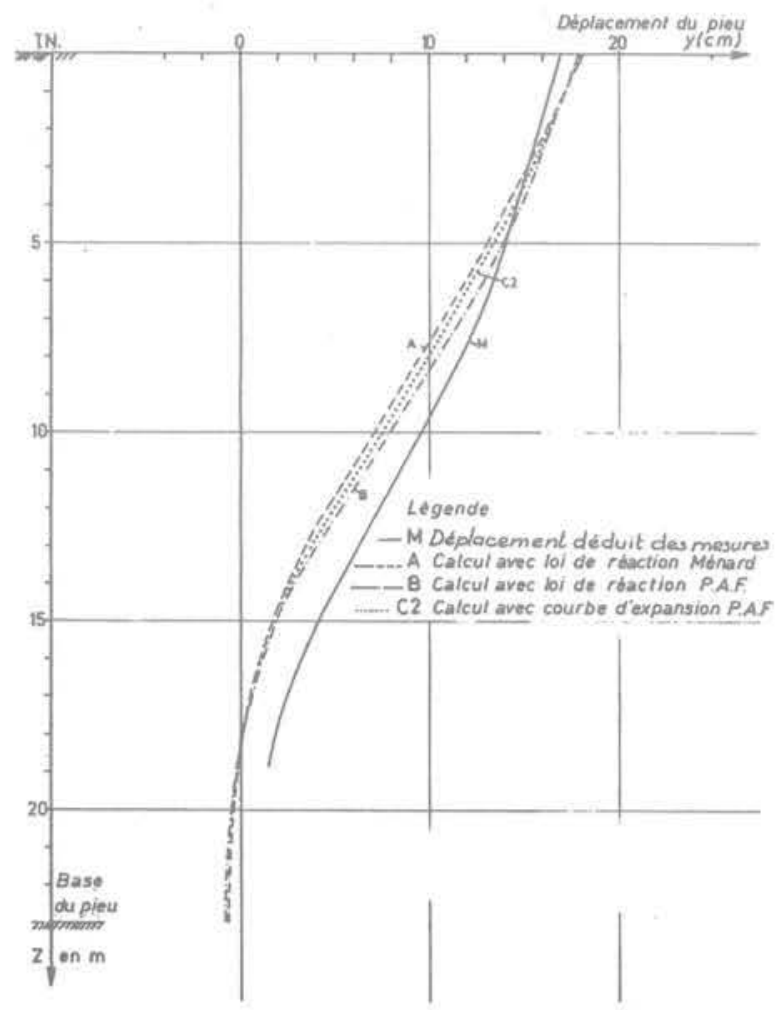

Fig. 21 Déplacements du pieu au 30.07.74 


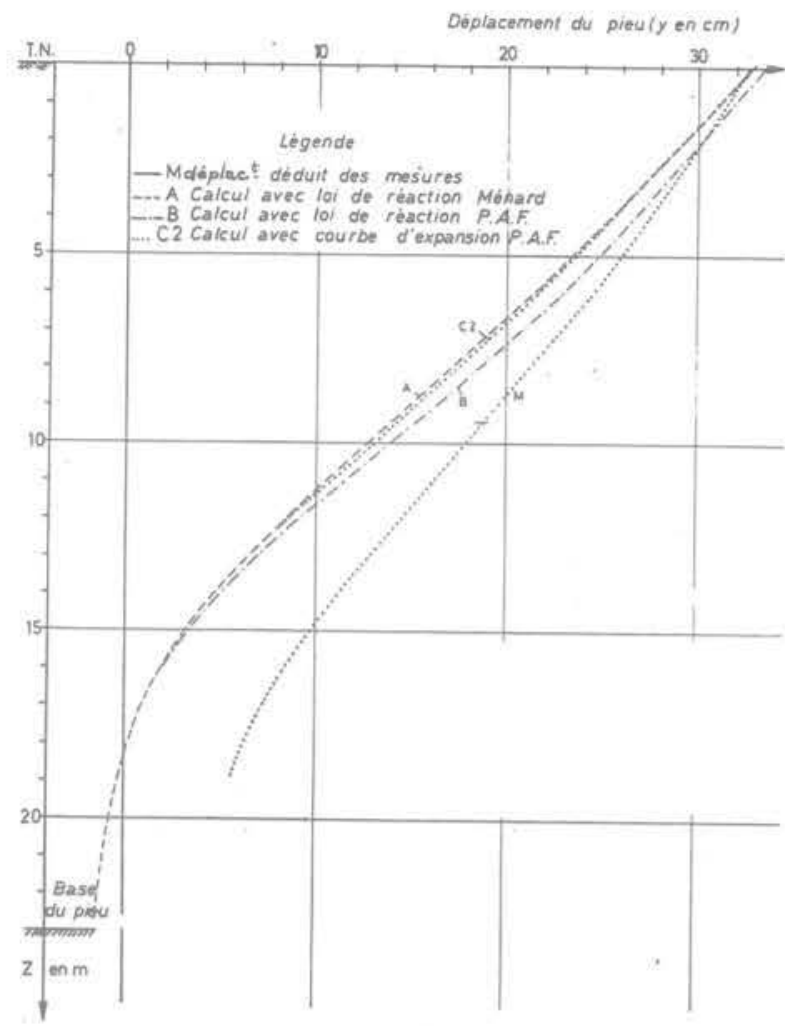

Fig. 22 Déplacements du pieu au 29.10.74
Par ailleurs, le déplacement $\mathrm{g}(\mathrm{z})$ étant donné et les lois de réactions dans le substratum étant les mêmes, on remarque que les moments croissent avec la raideur du sol dans la couche molle, ce qui est attendu. Si on se reporte à la figure 23 , on s'aperçoit, en effet, que pour les faibles déplacements, les lois $\mathrm{B}$ et $\mathrm{C}$ conduisent à des modules beaucoup plus élevés que la loi $\mathrm{A}$.

c) Les déplacements en tête, calculés, sont assez proches des déplacements mesurés. On remarque d'autre part que le rapport $y_{0} / g_{\max }$ est voisin de 1 , ce qui est cohérent avec l'étude paramétrique [13] (on a affaire à un pieu plutôt souple: $D / \ell_{0}$ est compris, pour la couche molle entre 2,1 et 2,4 .

Par contre, l'écart entre déplacements calculés et mesurés devient relativement important en pied. En dehors de l'influence de la loi de réaction et de la déformée du sol libre choisies, qui sera examinée plus loin, l'erreur possible sur la mesure de la rotation en tête joue un rôle non négligeable.

\section{Facteurs influençant les résultats des calculs}

Les résultats des calculs sont influencés principalement par :

- la loi de réaction du sol,

- la déformée du sol libre,

les conditions aux limites étant bien connues en tête et ayant peu d'influence en pied.

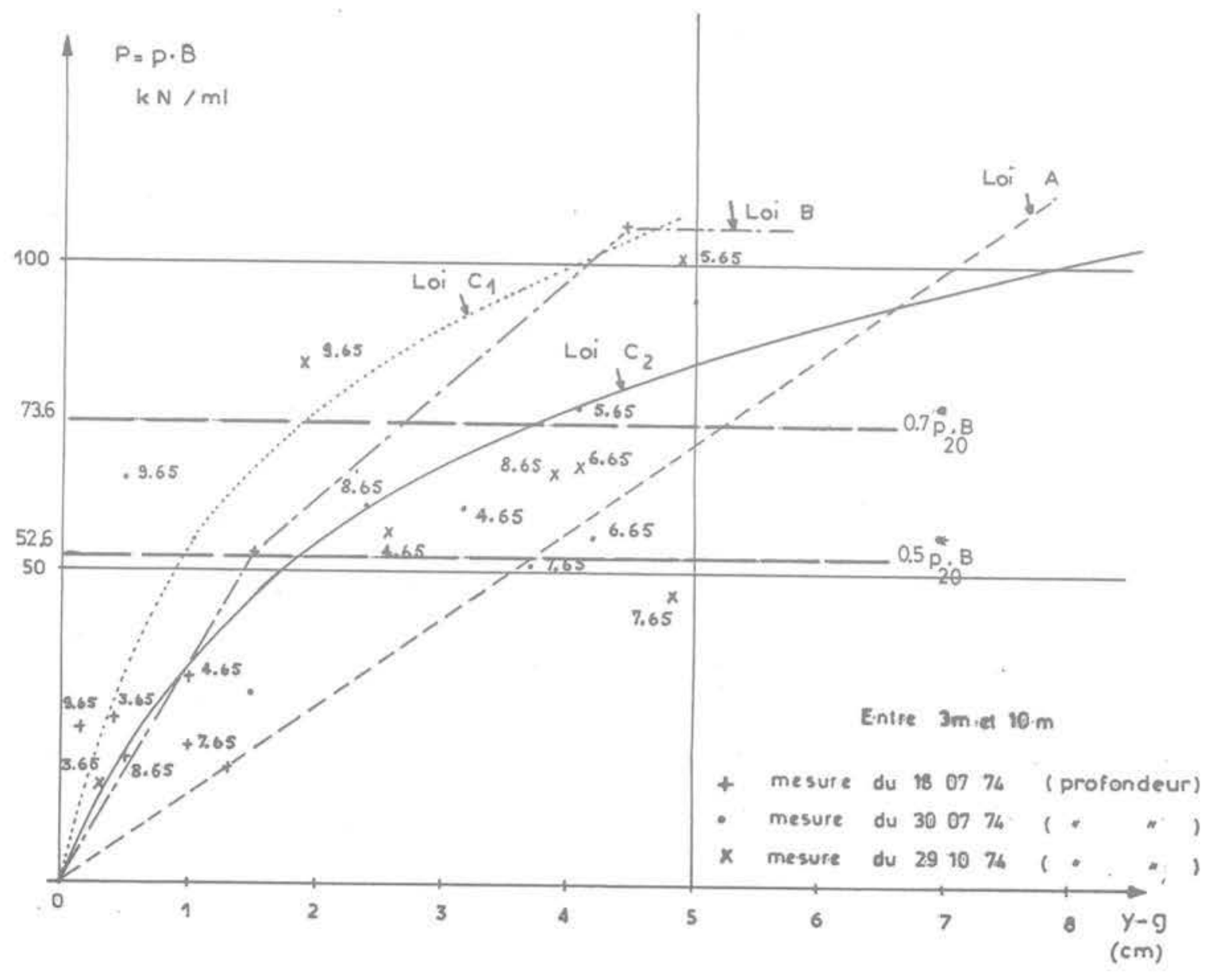

Fig. 23 Comparaison des lois de réaction pour les trois phases de chargement par le remblai 
Loi de réaction du sol

La loi de réaction A ( Ménard ) donne des moments plus faibles que les lois $B$ et $C_{2}$ (déduites du P. A. F.), la différence s'atténuant avec la charge. Parmi les courbes déduites du P. A. F. la " courbe de réaction " B donne des valeurs plus faibles des moments aux faibles charges que la "courbe d'expansion" $\mathrm{C}_{2}$; c'est l'inverse aux fortes charges.

Sur la figure 23 , on a reporté les 3 lois de réaction A, B, $C_{2}$ utilisées entre 3 (ou 4 ) et $11 \mathrm{~m}$ de profondeur et les points expérimentaux correspondant aux 3 phases, à différentes profondeurs. On a également reporté la loi $\mathrm{C}_{1}$.

Les points expérimentaux sont tirés des mesures par jauges en ce qui concerne $\mathrm{pB}$ et $\mathrm{y}$, et des courbes inclinométriques * corrigées (fig. 13) pour

$$
g(\Delta y=|y-g|) \text {. }
$$

Avec toute la prudence qui s'impose, compte tenu de l'incertitude sur les mesures de $g(z)$, on peut néanmoins constater que la loi $\mathrm{C}_{2}$ (P.A.F. avec déplacements doublés) est assez proche des courbes de réactions expérimentales, à condition de limiter la pression $p$ à une valeur comprise entre 0,5 et $0,7 p_{20}^{*}$, ce qui rejoint les recommandations déjà citées [12].

En ce qui concerne les moments à l'encastrement, $(15 \mathrm{~m})$, la valeur très forte donnée par les calculs peut provenir du module $\mathrm{E}_{\mathrm{s}}$ affecté à la couche comprise entre 14,50 et $17,50 \mathrm{~m}$ (sables et graviers). Ce module très fort $E_{s}=14608 \mathrm{kPa}$, est à comparer avec les rapports $\mathrm{pB} / \Delta \mathrm{y}$ tirés des mesures et qui donnent :

$$
\begin{array}{ll}
\text { à } 15 \mathrm{~m} & 5757 \mathrm{kPa} \\
\text { à } 16 \mathrm{~m} & 5096 \mathrm{kPa} \\
\text { à } 17 \mathrm{~m} & 2016 \mathrm{kPa} .
\end{array}
$$

II est possible que la seule mesure de module pressiométrique qu'on ait effectuée entre $14,50 \mathrm{~m}$ et $17,50 \mathrm{~m}\left(\mathrm{E}_{\mathrm{M}}=3600 \mathrm{kPa}\right.$ ) (fig. 2) ne représente pas le comportement réel de toute cette couche.

Pour étudier l'influence de la valeur du module dans le substratum, le calcul des moments maximaux a été repris avec un module $\mathrm{E}_{\mathrm{s}}=3963 \mathrm{kPa}$, uniforme entre $14,50 \mathrm{~m}$ et $20,50 \mathrm{~m}$, et avec les 3 lois de réaction $A, B$,
$C_{2}$ entre 0 et $14,50 \mathrm{~m}$. Les résultats sont donnés dans le tableau 7 dont les chiffres sont directement comparables à ceux du tableau 6. La comparaison de ces deux tableaux montre que le moment maximal dans le substratum est sensiblement réduit par correction des mesures du module éventuellement erronées. L'essai au P.A.F. qui conduit à des valeurs moins dispersées que l'essai “ Ménard " est, en ce sens, intéressant.

\section{Déformée libre du sol}

Si on n'a pas de critères précis pour évaluer le choix de la courbe $g(z)$, les comparaisons faites sur la figure 13 entre les courbes calculées et les courbes établies à partir des mesures (discutables, certes, comme on l'a vu plus haut mais néanmoins vraisemblables) paraissent acceptables.

II ne faut pas oublier toutefois que le moment maximal est proportionnel à $g_{\max }$, toutes choses égales par ailleurs : une erreur de $10 \%$ tout à fait possible dans la prévision de $\mathrm{g}_{\max }$, conduit à la même erreur relative sur le moment maximal.

Chargement horizontal du pieu en tête avant remblaiement $(\mathbf{3 0 . 0 4 . 7 4 )}$ (Phase 0)

II s'agit du chargement monotone de 0 à $120 \mathrm{kN}$ par paliers de $20 \mathrm{kN}$, l'effort horizontal étant appliqué à $0,20 \mathrm{~m}$ du sol.

On compare, sur les figures 24 et 25 , les moments et les déplacements mesurés et calculés pour le dernier palier (120 kN), les différents calculs étant faits avec les mêmes hypothèses sur les courbes de réaction du sol que précédemment, sauf pour la courbe « expansion P. A. F. " où les déplacements n'ont pas été doublés, le chargement étant, ici, " rapide " (loi $C_{1}$ ) [12].

Si on examine globalement les courbes de moments et de déplacements, on s'aperçoit que les courbes "calculées" et les courbes expérimentales ont des allures tout à fait semblables.

Si on se place du point de vue quantitatif, on constate que :

1) Pour les moments, et sauf en tête, (4 premiers

\begin{tabular}{|c|c|c|c|c|c|}
\hline & & $\begin{array}{l}\text { Calcul } \\
\text { loi } A^{*}\end{array}$ & $\begin{array}{l}\text { Calcul } \\
\text { loi B* }\end{array}$ & $\begin{array}{l}\text { Calcul } \\
\text { loi } \mathrm{C}_{2}{ }^{*}\end{array}$ & \multirow{7}{*}{$\begin{array}{l}\text { *au-delà de } 14,50 \mathrm{~m} \\
\text { c'est la loi } \mathrm{A} \\
\text { qui a été appliquée } \\
\text { dans les } 3 \text { cas, mais } \\
\text { avec un module constant } \\
\mathrm{E}_{\mathrm{s}}=3963 \mathrm{kPa} \\
\text { entre } 14,50 \mathrm{~m} \text { et } 20,50 \mathrm{~m} \text {. }\end{array}$} \\
\hline \multirow{3}{*}{$\begin{array}{c}\text { Maximum } \\
\text { couche } \\
\text { molle }\end{array}$} & Phase 1 & 0,33 & 0,70 & 0,76 & \\
\hline & Phase 2 & 0,45 & 0,87 & 0,74 & \\
\hline & Phase 3 & 0,90 & 1,37 & 1,14 & \\
\hline \multirow{3}{*}{$\begin{array}{c}\text { Moment } \\
\text { d'encastrement }\end{array}$} & Phase 1 & 0,73 & 0,86 & 0,93 & \\
\hline & Phase 2 & 0,90 & 1,005 & 1,03 & \\
\hline & Phase 33 & 1,05 & 1,17 & 1,12 & \\
\hline
\end{tabular}
mètres), la courbe expérimentale se situe entre les

Tableau 7

Rapport $\frac{\text { Moment maximal calculé }}{\text { Moment maximal mesuré }}$ 


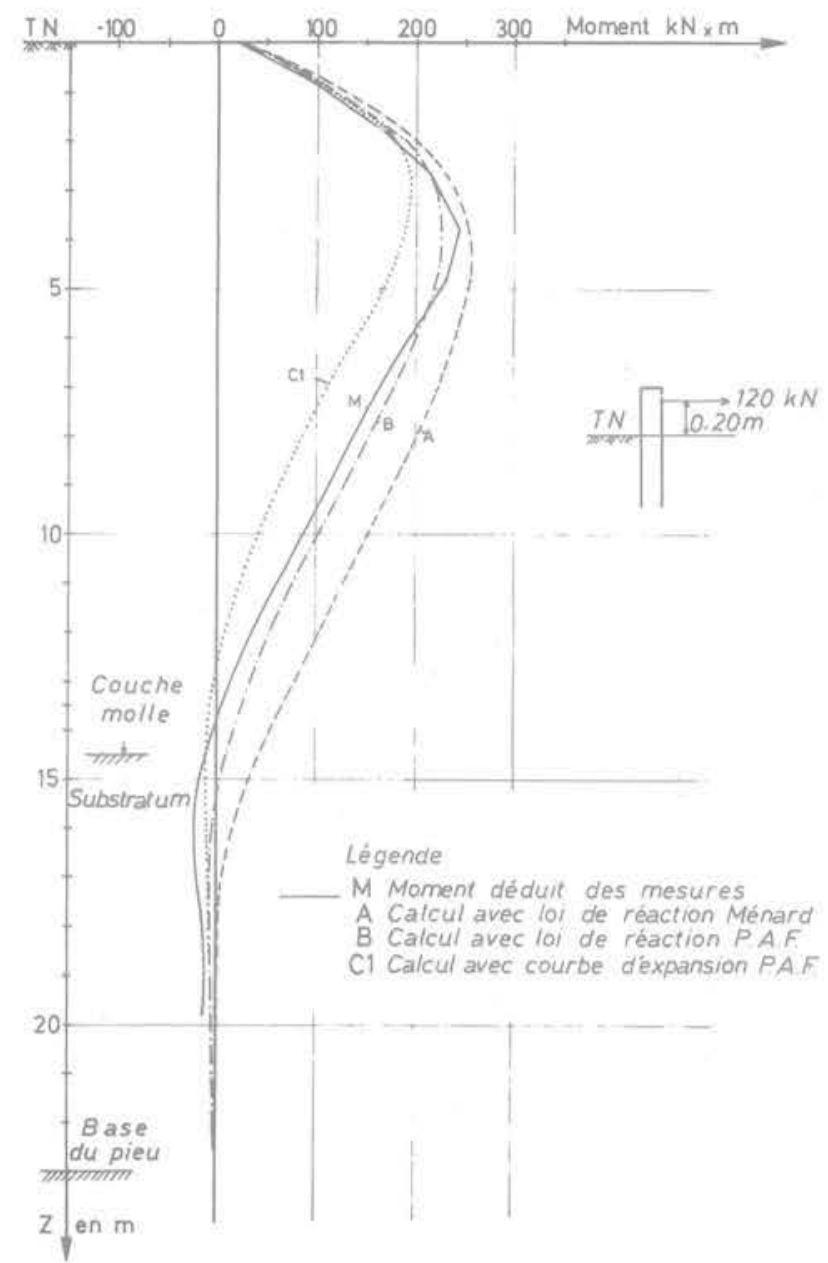

Fig. 24 Sollicitation horizontale en tête du 30,04.74: moments

courbes calculées par les lois A et $B$ au voisinage du maximum, et entre les courbes $B$ et $C_{1}$ dans la moitié inférieure de la couche molle.

2) Pour les déplacements, la courbe expérimentale se situe entre les courbes $B$ et $C_{1}$, la loi $A$ donnant globalement des déplacements trop forts (modules trop faibles).

Pour tirer des conclusions plus nettes, il est intéressant de comparer aux différentes profondeurs, les courbes de réaction $A, B$, et $C_{1}$ et la courbe expérimentale. C'est ce qui est fait sur la figure 26 .

On a reporté sur les 4 premiers graphiques, pour chacun des niveaux $0,0,75 \mathrm{~m}, 1,75 \mathrm{~m}, 2,75 \mathrm{~m}$, la courbe expérimentale et les courbes de réaction $\mathrm{A}, \mathrm{B}$, $\mathrm{C}_{1}$ utilisées dans les calculs, mais frappées des coefficients minorateurs de la pression $\left(1+z / z_{c}\right) / 2$ dont les valeurs respectives sont $0,5,0,6875,0,9375$ et 1, ainsi que la courbe expérimentale au même niveau. Le $5^{\circ}$ graphique donne les courbes de réaction $\mathrm{A}, \mathrm{B}, \mathrm{C}_{1}$ utilisées pour les calculs entre 3 (ou 4 ) et $11 \mathrm{~m}$, ainsi que les points expérimentaux, pour différentes profondeurs, correspondant à la charge finale de $120 \mathrm{kN}$ en tête. La faiblesse des déplacements pour les étapes intermédiaires du chargement n'a pas permis de tracer une courbe de réaction significative à chaque niveau, pour cette couche de 3 à $11 \mathrm{~m}$.

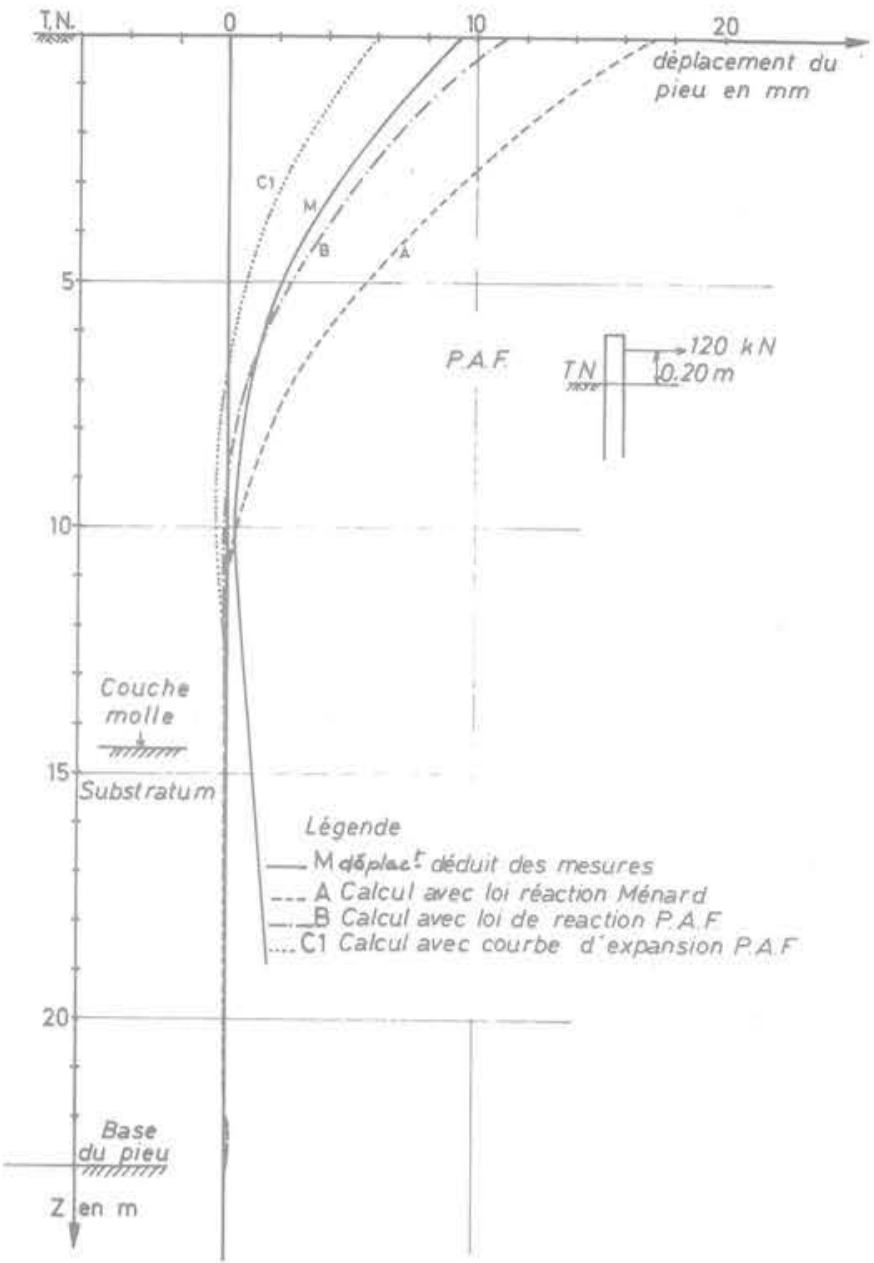

Fig. 25 Sollicitation horizontale en tête du 30.04.74: déplacements

L'examen de ces graphiques conduit aux conclusions suivantes :

1) Pour les zones proches de la surface (jusqu’à $1,75 \mathrm{~m})$, la courbe de réaction expérimentale coïncide avec la courbe A (Ménard), la courbe B et surtout la courbe $C_{1}$ étant beaucoup trop raides.

2) En profondeur (à partir de $2,75 \mathrm{~m}$ ), la courbe $\mathrm{A}$ donne des modules trop faibles et les courbes de réaction expérimentales correspondent plutôt aux courbes tirées du P.A.F. (B et même $C_{1}$, et cette dernière pour la plupart des points de la couche 3-11 m).

On comprend alors pourquoi les résultats des figures 24 et 25 montrent que, "globalement $n$, c'est la loi B qui se rapprochie le plus des résultats expérimentaux.

\section{Conclusion}

Sur le plan expérimental, il apparaît que la mesure des moments à partir des mesures d'élongations des jauges a donné satisfaction. Par contre, le problème majeur a été la mesure des déplacements libres du sol. Depuis 1974, fort heureusement, de grands progrès ont été réalisés dans le domaine des mesures par inclinomètre [14] [15], ce qui permet de penser que des 


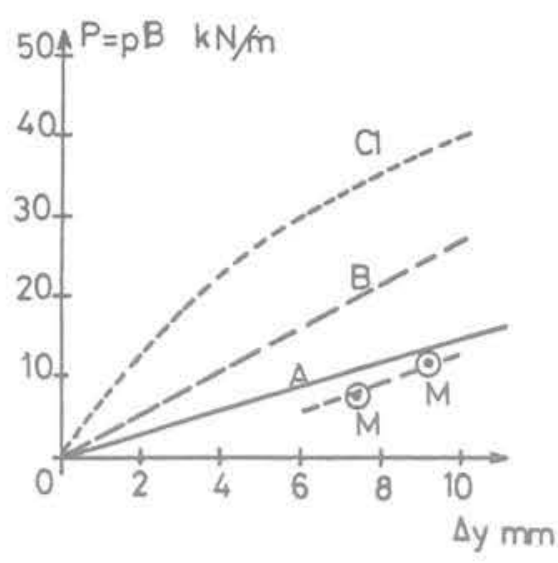

1) $Z=0$

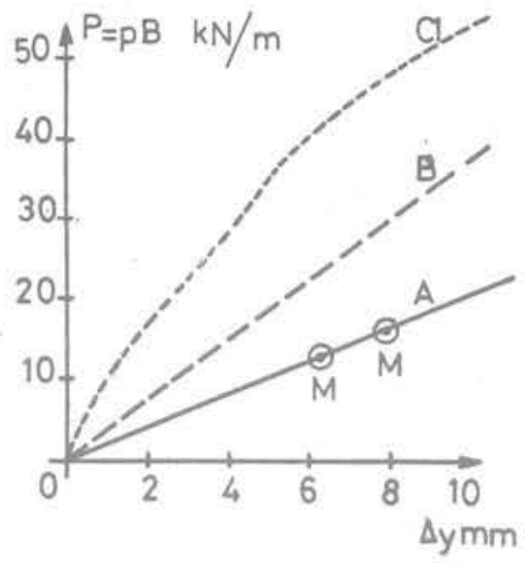

2) $Z=0.75 \mathrm{~m}$

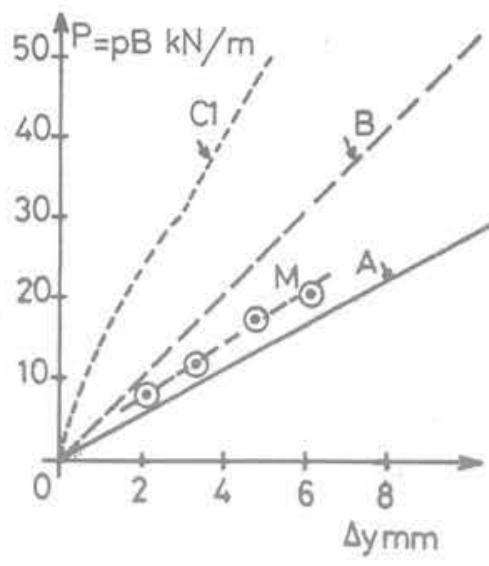

3) $Z=1,75 \mathrm{~m}$

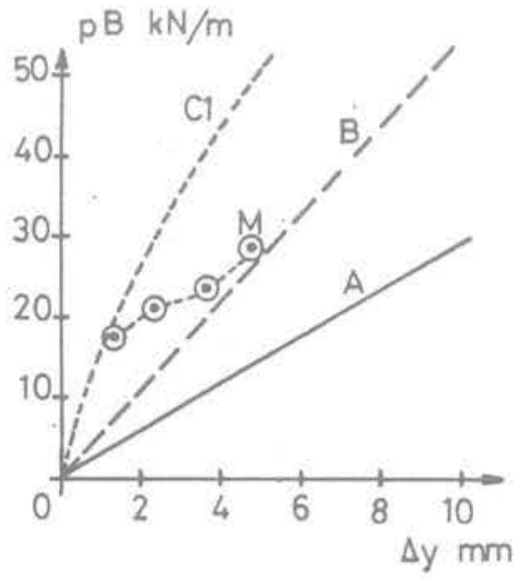

4) $Z=2.75 \mathrm{~m}$

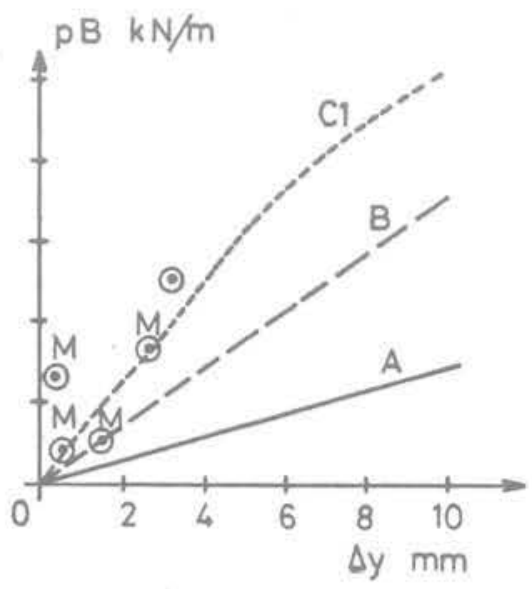

5) $\mathrm{Z}=3 \mathrm{a} 11 \mathrm{~m}$

Fig. 26 Chargement horizontal en tête. Courbes de réactions aux différents niveaux

A calcul loi de Ménard

$B$ calcul loi de réaction PAF

$C_{1}$ calcul courbe d'expansion PAF (déplacements non doublés)

$M$ mesures

expérimentations futures de pieux soumis à des poussées latéralés de sol se dérouleront dans de meilleures conditions sur ce point.

Sur le plan théorique et de la méthode de calcul proposée, les comparaisons des calculs et des résultats expérimentaux montrent clairement que cette méthode permet de prendre en compte les aspects déterminants du mécanisme de pieux soumis à des poussées latérales. Cette méthode nécessite encore un certain calage sur l'expérience des paramètres à introduire. On notera toutefois qu'elle donne déjà, en son état actuel, des résultas fort satisfaisants pour le cas de ce pieu de Provins. La comparaison des résultats avec différentes lois de réaction, montre que la loi Ménard, conduit à des moments trop faibles dans la couche molle et met en avant l'intérêt des résultats obtenus avec le pressiomètre autoforeur (P.A.F.) dans $c \theta$ genre de sols. II faut relever néanmoins que le moment déterminant pour le dimensionnement du pieu traité ici est le moment.dans le substratum à la phase 3 pour lequel l'essai Ménard conduit à des résultats plus proches des valeurs mesurées.

Enfin, il apparaît que les recherches futures, outre la poursuite des mesures de déplacements horizontaux à proximité de remblais afin de préciser la méthode de détermination de $\mathrm{g}(\mathrm{z})$, devront également examiner l'effet du temps sur les lois de réaction à prendre en compte. 


\section{Références bibliographiques}

[1] G. Bigot, F. Bourges, R. Frank et Y. Guegan (1977), "Action du déplacement latéral du sol sur un pieu ». C. R. du $9^{\circ}$ Congrès Int. Méc. Sols et Trav. Fond. Tokyo, vol. 1, pp. 407-410 et Bull. Liaison L. P. C. Spécial VI. F, pp. 59-63 (français) et Spécial VI. E, pp. 62-66 (anglais).

[2] F. Bourges, R. Frank et C. Mieussens (1980), "Calcul des efforts et des déplacements engendrés par des poussées latérales sur les pieux $\%$. Note technique Département des Sols et Fondations, L.C.P.C.

[3] G. Bigot, F. Bourges et R. Frank (1981), "Étude expérimentale d'un pieu soumis aux poussées latérales du sol n. Rapport de Recherches. Département Sols et Fond., Lab. Centr. des P. et $\mathrm{CH}_{\text {., }} 73$ pp.

[4] M. Diruy (1973), "Extensomètre à réseaux, type L.C.P.C.». Bull. Liaison L.P.C. $n^{\circ} 64, p p, 46-52$.

[5] F. Baguelin et J. F. Jézéquel (1972), «Étude expérimentale du comportement des pieux sollicités horizontalement ". Bull. Liaison L. P. C. $n^{\circ} 62$, pp. 129170.

[6] H. Matlock, E. Ripperger (1956), « Procedures and instrumentation for tests on a laterally loaded pile". Proc. 8th Texas Conf. On soil Mech. an Found. Eng. Research University of Texas.

[7] H. G. Poulos (1973), «Analysis of piles in soil undergoing lateral movement ». AM. Soc. Civ. Eng., Jal Soil Mech. Fdn. Div., SM5, May, pp. 391-406.
[8] R. Marche (1973), "Lateral pressure of clayed soils on structures". Session spéciale $n^{\circ} 5$, C.R. du $8^{\circ}$ Congrès Int. Méc. Sols et Trav. Fond., Moscou, vol. 4-3, pp. 247-252.

[9] F. Baguelin, R. Frank et Y. Guegan (1976), "Calcul sur ordinateur des pieux sollicités horizontalement ou subissant des poussées parasites». Bulletin Liaison L. P.C. $n^{\circ} 84$, pp. $113-120$.

[10] F. Bourges et C. Mieussens (1979), "Déplacements latéraux à proximité des remblais sur sols compressibles. Méthode de prévision ». Bull. Liaison L.P.C. $n^{\circ} 101$, pp. 73-100.

[11] F. Tavenas, C. Mieussens et F. Bourges (1979) "Lateral displacements in clay foundations under embankments ». Revue Canadienne de Géotechnique, vol. $16, n^{\circ} 3$, pp. $532-550$.

[12] S. Amar, F. Baguelin, R. Frank, J.-F. Jézéquel (1981), «L'autoforage . Travaux $n^{\circ} 552$, pp. 63-75.

[13] R. Frank, M. Kutniak (1981), "Étude par la méthode du module de réaction, des pieux soumis à des poussées latérales ». Rapport de recherche L. P. C. n 107, Décembre 1981.

[14] P. Ducasse et M. Virollet (1977), « Amélioration de la représentativité de l'essai clinométrique $n$. Rapport du L.R. de Toulouse.

[15] B. Pincent (1977), « Surveillance des glissements de terrain $»$. Revue Française de Géotechnique $n^{\circ} 1$, pp. 93-104. 
